\title{
19. OCEANIC BASALTS FROM THE TYRRHENIAN BASIN, DSDP LEG 42A, HOLE 373A
}

\author{
Volker Dietrich, Institut für Kristallographie und Petrographie, ETH Zürich, Switzerland, \\ Rolf Emmermann ana Harald Puchelt, Institut für Petrographie und Geochemie der Universität, Karlsruhe, \\ Germany, \\ and \\ Jörg Keller, Mineralogisches Institut der Universität, Freiburg/Br., Germany
}

\begin{abstract}
During Leg 42A, drilling in Hole 373A in the Central Tyrrhenian Sea penetrated Miocene basaltic basement for 190 meters and recovered basalt from up to 450 meters below the sea floor. Despite mineralogical and chemical alteration due to hydrothermal processes in the Tyrrhenian marginal basin, petrological analogies to high-Al abyssal basalts can be traced.

Two types of interlayered basalt were recognized: (a) a lower $\mathrm{Ti}$ group (Core 5, Sections 1-2; Cores 7 and 10), and (b) a higher Ti group (Core 5, Section 3; Cores 11 and 12; and outer barrel). We distinguish two magma series: a more primitive magma leading to the plagioclase-bearing low-titanium basalts with affinities to high$\mathrm{Al}$ abyssal tholeiites, and a more differentiated magma leading to the basalts with higher titanium content.
\end{abstract}

\section{INTRODUCTION}

During DSDP Leg 42A, basalt was recovered from Hole $373 \mathrm{~A}$ in the abyssal plain of the Central Tyrrhenian Sea (Figure 1). Basaltic breccia was first encountered under a Quaternary and Pliocene sediment cover about 270 meters below the sea floor. Then it was drilled 190 meters through a basaltic sequence of breccia and lava flows and took 11 cores at depths as indicated on Figure 2.

Site $373 \mathrm{~A}$ is on the lower flanks of one of the large Tyrrhenian seamounts (Figure 1) and the basalts belong to the acoustic basement (Site 373A report, this volume). Assuming that the age of the overlying sediments is its upper limit, the basaltic, basement is probably lower Pliocene/upper Miocene (Barberi et al., 1977, Kreuzer et al., 1977).

\section{GEODYNAMICS OF THE TYRRHENIAN SEA}

The formation of small ocean basins in the western Mediterranean has caused considerable discussion (see Ritsema, 1970) since the discovery of their youthful age and the nature (oceanic to transitional) of the crust and mantle beneath became evident (Menard, 1967). Early geological work in the borderlands of the Tyrrenian Sea had revealed the existence of a former landmass in the area presently occupied by the basin. Evidence of currents and clastic sedimentary components in the sedimentary sequence point to the existence of such a landmass until Miocene time. Following the earlier concept of the tectonic break-up of a Tyrrhenian continent, several other concepts involving complicated oceanization models were presented. These are reviewed by Ritsema (1970).

The plate tectonics concept invokes spreading processes with formation of new basaltic crust in widening basins. In this context Boccaletti and Guazzone (1972), Dewey et al. (1973), and Barberi et al. (1973) have compared the Tyrrhenian Basin with formerly spreading but now inactive marginal basins which nevertheless still have high heat flow values (Karig, 1971).

The deep-focus earthquakes which occur in the southeastern Tyrrhenian Sea and the calcalkaline magmatism which occurs in the Aeolian Islands are characteristic of an active island arc (Barberi et al., 1973; Keller, 1974). The age of the exposed island arc volcanic rocks, however, is less than 1 m.y.B.P. (Barberi et al., 1974), whereas the Tyrrhenian Basin formed about 10 m.y. ago. The high heat flow values and a rather indistinct pattern of magnetic anomalies in the Tyrrhenian Basin suggest that it was a spreading marginal basin (Ryan et al., 1970). But the pattern of the Tyrrhenian spreading centers that can be inferred from the magnetic anomalies does not exhibit any simple regularity. The picture is certainly complicated by the rotation of individual crustal blocks such as Sardinia-Corsica, the Italian penisula, and smaller microplates (Alvarez et al., 1974; Lowrie and Alvarez, 1974). Spreading centers may have developed between such rotating blocks and the existence of areas with continental crust within such a domain is possible. (Honnorez and Keller, 1968; Heezen et al., 1971).

The main purpose of drilling in the Tyrrhenian basement was to test whether the chemical characteristics of abyssal basalts, formed at oceanic spreading centers or their marginal basin analogs, are present. If so, we could conclude that upper mantle processes similar to those occurring under mid-ocean ridges have acted as well, in the Tyrrhenian Basin.

Dredge samples of basalt from the Tyrrhenian seamounts (mainly from Mount Marsili) were availa- 


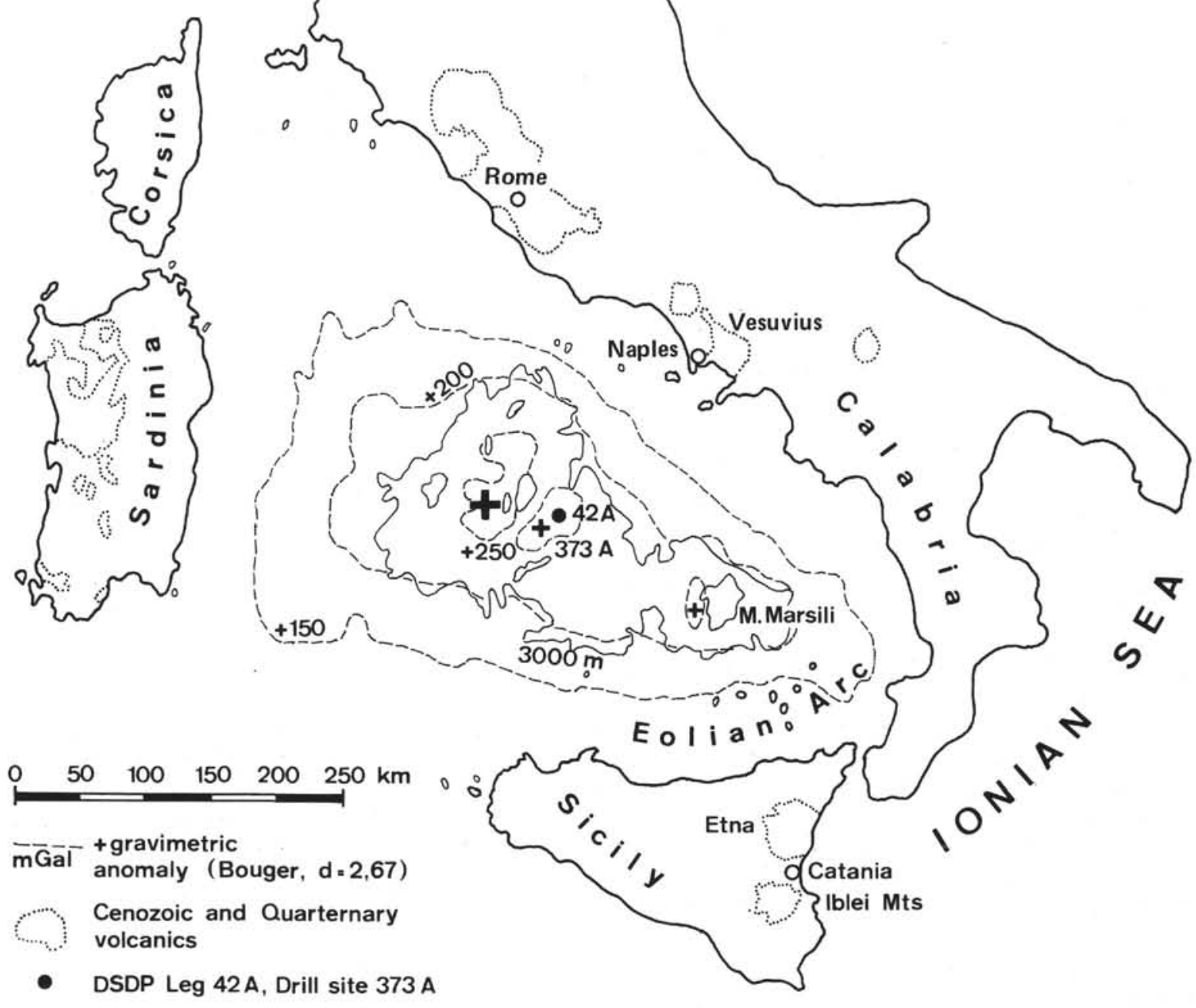

Figure 1. The Tyrrhenian Basin.

ble for study (Maccarone, 1970; Del Monte, 1972; Keller and Leiber, 1974) (Figure 1). These basalt samples are mostly alkaline. No data were published to support a more detailed characterization (for example, designation as "abyssal tholeiite") for there were few samples with tholeiitic affinity (e.g., No. 29M of Del Monte, 1972). Nevertheless, such designation was widely quoted to support the existence of spreading precesses within the Tyrrhenian Sea. Moreover, seamount volcanism apparently occurred much later than basin formation and lasted until the upper Quaternary (Keller and Leiber, 1974).

\section{PETROGRAPHIC DESCRIPTION OF ANALYZED SAMPLES}

Most basalt samples are well crystallized and have intergranular textures of fresh plagioclase, clinopyroxene, and titanomagnetite in a matrix of smectite, chloritic minerals, or both. This matrix constitutes up to $30 \%$ of the rock volumes. X-ray analyses, with use of ethylene glycol treatment, on three samples showed the typical shift of the 100 montmorillonite peak from 14 to $18 \AA$. A chlorite + montmorillonite mixture is abundant in two of the samples $(7-2,136-140 \mathrm{~cm}$, and 7-3, 111$112.5 \mathrm{~cm}$ ). Some smectite patches, clearly pseudomorphs after olivine and very rarely occurring olivine relics are preserved (e.g., 7-4, 135-140 cm, and 7-5, 10$14 \mathrm{~cm}$ ). However, the extent of original modal olivine remains uncertain.

Two petrographic types of basalt are recognized; (1) plagioclase phyric and (2) aphyric to sparsely microphyric. This distinction corresponds to a chemical grouping explained below (Table 4).

Plagioclase phenocrysts are calcic with compositions ranging up to $\mathrm{An}_{84}$. Groundmass plagioclase ranges mainly from $\mathrm{An}_{50-60}$ (Figure 3, Table 1). Along with 


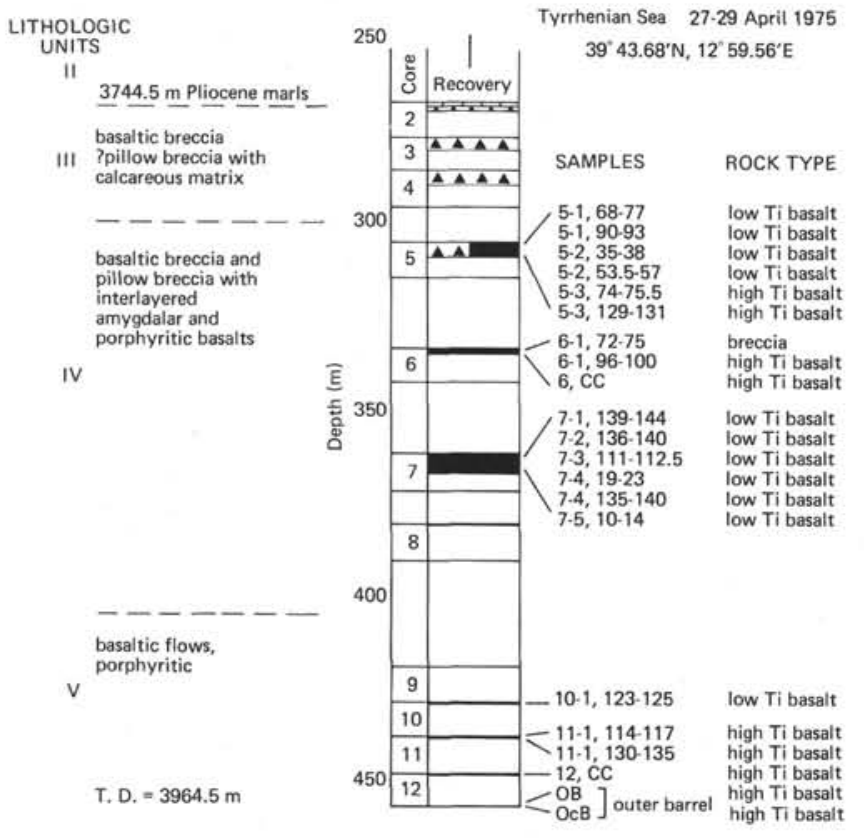

Figure 2. Graphic lithology of Site 373A. The drilling started at a depth of 96.5 meters sub-bottom within Quaternary Nannofossil marls (lithologic Unit I; Site $373 A$ Report, this volume). At 270 meters, Glomar Challenger drilled into basaltic breccias (containing pillow fragments) associated with lava flows. The drilling was terminated at 457.5 meters in basaltic lava flows. Twenty basaltic samples from six cores out of Units IV and $V$ were analyzed.

these groundmass labradorites, almost pure albite (with an increased orthoclase component) has been found by microprobe analysis. This suggests the beginning of a process of mineralogical readjustment and chemical exchange which is more pronounced in true ocean-floor metamorphic spilites (Cann, 1969).

Chlorite and an unidentified green amphibole-like mineral (riebeckite?) developed as small rims surrounding the groundmass clinopyroxene and as tiny, but discrete, crystals within the chlorite/smectite patches. X-ray powder data from Sample 7-4, 10-4 cm, confirmed the existence of chlorite. Thus, the first indication of a change toward a greenschist facies mineralogy as described for oceanic basalts by Miyashiro et al. (1971) is apparent.

In general, however, groundmass clinopyroxenes are fresh. Their composition (Table 2, Figure 4) is salitic and similar to the clinopyroxenes reported by Ridley et al. (1974). Iron enrichment is present but not pronounced. No Ca-poor pyroxene has been detected.

Calcite occurs as veinlets, vesicle fillings, and patches within the groundmass and plagioclase phenocrysts. All samples analyzed contain only minor amounts of calcite. Zeolites replace plagioclase and fill vesicles in the most altered samples, but these are not suitable for chemical analysis $(5-1,68-71 \mathrm{~cm} ; 5-2,35-$ $38 \mathrm{~cm}$; and 6-1, 133-136 cm).

\section{GEOCHEMICAL METHODS AND DISCUSSION OF RESULTS}

Twenty samples were analyzed by X-ray fluorescence, atomic absorption, ionic neutron activation, and wet chemical methods to determine their major and trace element contents. All major element determinations were made at least three times by each of the different laboratories involved (Freiburg i.Br., Karlsruhe, Zïrich, and Canberra). The results given in Table 3 are in good agreement. Determinations were carried out by:

a) XRF for $\mathrm{SiO}_{2}, \mathrm{TiO}_{2}, \mathrm{Al}_{2} \mathrm{O}_{3}, \mathrm{Fe}_{2} \mathrm{O}_{3}$ (total), $\mathrm{MnO}, \mathrm{MgO}, \mathrm{CaO}$, and $\mathrm{K}_{2} \mathrm{O}$;

b) AAS for $\mathrm{Na}_{2} \mathrm{O}, \mathrm{K}_{2} \mathrm{O}, \mathrm{CaO}, \mathrm{MgO}, \mathrm{Fe}_{2} \mathrm{O}_{3}$ (total), and $\mathrm{Al}_{2} \mathrm{O}_{3}$;

c) INAA for $\mathrm{Na}_{2} \mathrm{O}, \mathrm{K}_{2} \mathrm{O}, \mathrm{CaO}, \mathrm{MnO}$, and $\mathrm{Fe}_{2} \mathrm{O}_{3}$ (total);

d) Wet chemical methods for $\mathrm{SiO}_{2}, \mathrm{TiO}_{2}, \mathrm{FeO}$, $\mathrm{P}_{2} \mathrm{O}_{5}, \mathrm{H}_{2} \mathrm{O}^{+}$, and $\mathrm{CO}_{2}$;

e) Electron microprobe analysis (by J. Keller) on plagioclase and pyroxene phenocrysts.

All samples were analyzed for the following elements by INAA, but these elements were below the detection limits indicated:

$\mathrm{Sb}(<1 \mathrm{ppm}), \mathrm{Ta}(<1 \mathrm{ppm}), \mathrm{U}(<1.5 \mathrm{ppm}), \mathrm{Th}(<1$ $\mathrm{ppm})$, and $\mathrm{Cs}(<2 \mathrm{ppm})$. The trace element data are included in Table 3. the methods applied were: (a) $\mathrm{XRF}$ for $\mathrm{Rb}, \mathrm{Sr}, \mathrm{Zr}, \mathrm{Y}, \mathrm{Pb}$, and U; (b) AAS for $\mathrm{Li}, \mathrm{Sr}$, and $\mathrm{Cu}$; (c) INAA for $\mathrm{Sc}, \mathrm{Cr}, \mathrm{Co}, \mathrm{Hf}, \mathrm{La}, \mathrm{Ce}, \mathrm{Sm}, \mathrm{Eu}$, $\mathrm{Tb}, \mathrm{Dy}, \mathrm{Yb}$, and $\mathrm{Lu}$
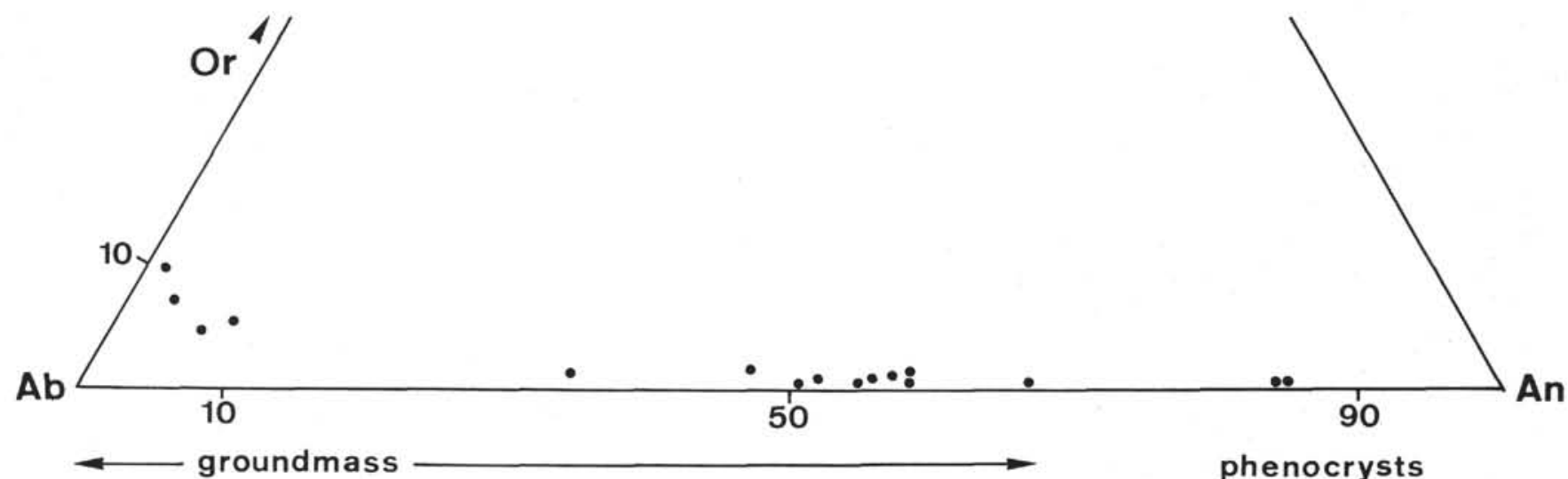

Figure 3. Plagioclase plot for Hole $373 \mathrm{~A}$ basalts. 
TABLE 1

Chemical Composition and Structural Formula of Phenocrystic and Groundmass Plagioclases from 373A Basalts (microprobe analyses)

\begin{tabular}{|c|c|c|c|c|c|c|c|c|c|c|c|c|c|c|c|c|c|}
\hline & \multicolumn{5}{|c|}{$5-1,90-93 \mathrm{~cm}$} & \multicolumn{2}{|c|}{$5-2,53.5-57 \mathrm{~cm}$} & \multirow{2}{*}{$\begin{array}{l}\text { 6, CC } \\
\text { Grdm. }\end{array}$} & \multicolumn{2}{|c|}{$7-1,139-144 \mathrm{~cm}$} & \multicolumn{2}{|c|}{$11-1,130-135 \mathrm{~cm}$} & \multicolumn{5}{|c|}{$12, \mathrm{CC}$} \\
\hline & Grdm. & Pheno. & Grdm. & Grdm. & Grdm. & Grdm. & Grdm. & & Pheno. & Grdm. & Grdm. & Grdm. & Grdm. & Grdm. & Grdm. & Grdm. & Grdm. \\
\hline $\mathrm{SiO}_{2}$ & 56.66 & 45.75 & 50.77 & 54.88 & 58.61 & 54.56 & 65.16 & 52.84 & 45.97 & 53.11 & 53.42 & 53.65 & 53.10 & 53.32 & 66.22 & 66.75 & 67.76 \\
\hline $\mathrm{Al}_{2} \mathrm{O}_{3}$ & 27.02 & 34.72 & 30.97 & 28.56 & 25.06 & 28.28 & 20.55 & 29.46 & 34.29 & 29.32 & 29.13 & 29.02 & 29.83 & 29.19 & 20.61 & 20.08 & 18.90 \\
\hline $\mathrm{FeO}$ total & 0.86 & 0.24 & 0.6 & 0.99 & 1.06 & 0.90 & 1.27 & 0.68 & 0.33 & 0.63 & 1.67 & 0.66 & 1.43 & 0.64 & 0.42 & 0.57 & 0.75 \\
\hline $\mathrm{CaO}$ & 9.05 & 17.46 & 13.71 & 9.44 & 7.15 & 10.57 & 1.71 & 12.15 & 17.38 & 11.82 & 11.08 & 11.35 & 10.19 & 11.54 & 1.32 & 0.65 & 0.31 \\
\hline $\mathrm{Na}_{2} \mathrm{O}$ & 6.41 & 1.70 & 3.85 & 5.82 & 7.62 & 5.53 & 10.19 & 4.77 & 1.75 & 4.89 & 4.44 & 5.17 & 5.28 & 5.13 & 10.55 & 10.68 & 10.53 \\
\hline $\mathrm{K}_{2} \mathrm{O}$ & & 0.13 & 0.09 & 0.31 & 0.32 & 0.16 & 1.00 & 0.09 & 0.15 & 0.23 & 0.26 & 0.16 & 0.17 & 0.19 & 0.88 & 1.26 & 1.76 \\
\hline \multicolumn{18}{|l|}{320} \\
\hline $\mathrm{Si}$ & 10.203 & 8.437 & 9.269 & 9.920 & 10.541 & 9.883 & 11.568 & 9.560 & 8.483 & 9.649 & 9.719 & 9.731 & 9.639 & 9.682 & 11.679 & 11.778 & 11.965 \\
\hline Al & 5.733 & 7.546 & 6.663 & 6.085 & 5.312 & 6.038 & 4.300 & 6.315 & 7.458 & 6.279 & 6.239 & 6.203 & 6.383 & 6.247 & 4.284 & 4.177 & 3.933 \\
\hline $\mathrm{Fe}$ & 0.129 & 0.037 & 0.092 & 0.150 & 0.159 & 0.137 & 0.189 & 0.104 & 0.050 & 0.096 & 0.253 & 0.099 & 0.217 & 0.097 & 0.062 & 0.085 & 0.111 \\
\hline $\mathrm{Ca}$ & 1.746 & 3.451 & 2.682 & 1.827 & 1.378 & 2.051 & 0.325 & 2.368 & 3.436 & 2.301 & 2.158 & 2.206 & 1.983 & 2.244 & 0.250 & 0.124 & 0.059 \\
\hline $\mathrm{Na}$ & 2.238 & 0.609 & 1.364 & 2.041 & 2.657 & 1.942 & 3.509 & 1.682 & 0.628 & 1.721 & 1.566 & 1.817 & 1.857 & 1.807 & 3.608 & 3.655 & 3.604 \\
\hline $\mathrm{K}$ & & 0.031 & 0.022 & 0.071 & 0.074 & 0.037 & 0.226 & 0.020 & 0.035 & 0.053 & 0.060 & 0.037 & 0.040 & 0.043 & 0.198 & 0.284 & 0.396 \\
\hline & 20.049 & 20.111 & 20.092 & 20.094 & 20.121 & 20.088 & 20.117 & 20.049 & 20.090 & 20.099 & 19.995 & 20.093 & 20.119 & 20.120 & 20.081 & 20.103 & 20.068 \\
\hline $\mathrm{Na}:$ & 56.2 & 14.9 & 33.5 & 51.8 & 64.4 & 48.2 & 86.4 & 41.3 & 15.3 & 42.2 & 41.4 & 44.8 & 47.9 & 44.1 & 89.0 & 90 & 88.8 \\
\hline K: Mol \% & 0 & 0.8 & 0.5 & 1.8 & 1.8 & 0.9 & 5.6 & 0.5 & 0.8 & 1.3 & 1.6 & 0.9 & 1.0 & 1.0 & 4.9 & 7 & 9.8 \\
\hline $\mathrm{Ca}:$ & 43.8 & 84.4 & 65.9 & 46.4 & 33.5 & 50.9 & 8.0 & 58.2 & 83.8 & 56.5 & 57.0 & 54.3 & 51.1 & 54.8 & 6.2 . & 3 & 1.4 \\
\hline
\end{tabular}

TABLE 2

Chemical Composition and Structural Formula of Groundmass Clinopyroxenes from 373A Basalts (microprobe analyses)

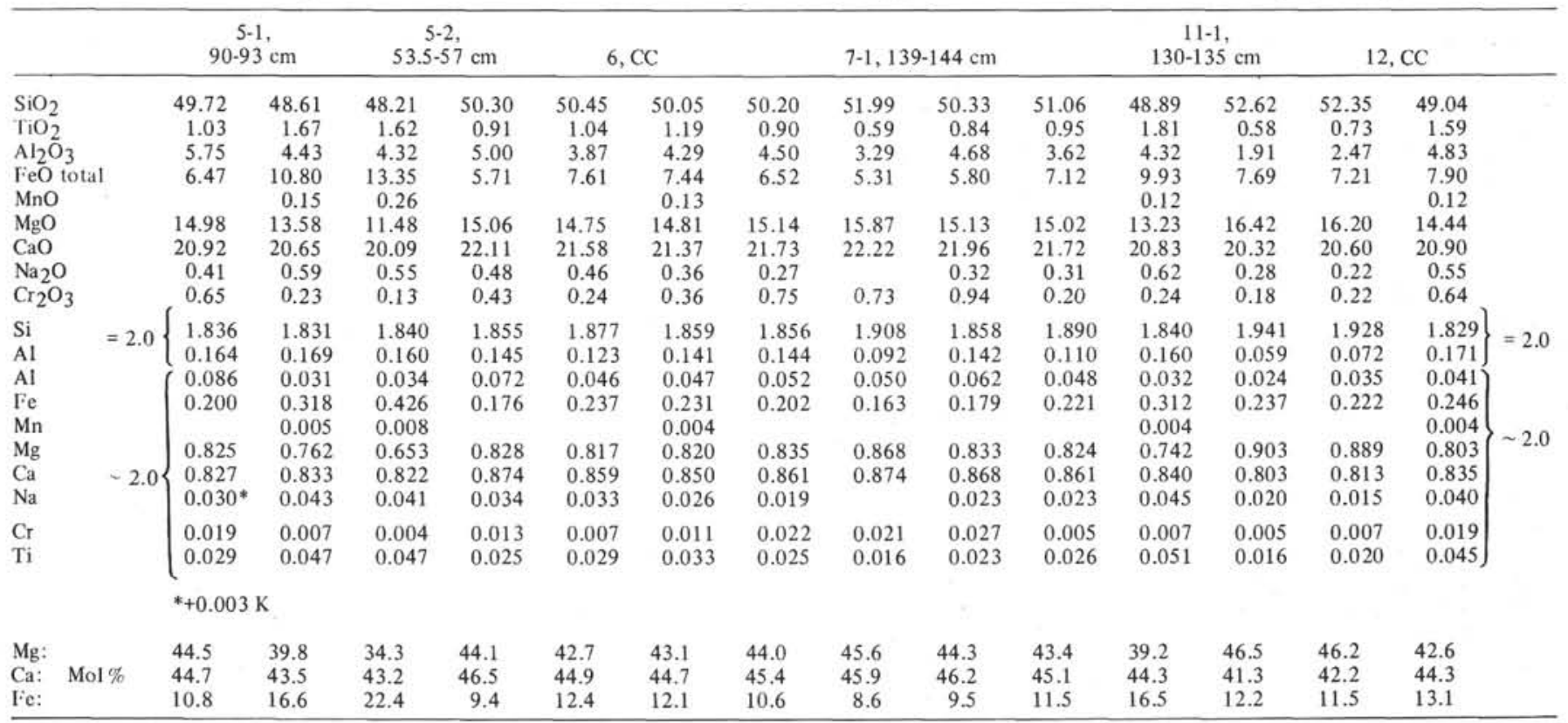

To check accuracy, standard reference samples were also analyzed. The values obtained for USGS-BCR-1 in the Karlsruhe laboratory are given by Puchelt et al. (1976) in the Initial Reports of the Deep Sea Drilling Project, Volume 37.

In addition, the abundance of a large number of trace elements was determined by XRF, AAS, and INAA. A few samples were tested by Spark Source Mass Spectroscopy for $\mathrm{Rb}, \mathrm{Sr}, \mathrm{Ba}, \mathrm{Pb}, \mathrm{La}, \mathrm{Ce}, \mathrm{Pr}, \mathrm{Nd}$, $\mathrm{Sm}, \mathrm{Eu}, \mathrm{Gd}, \mathrm{Tb}, \mathrm{Dy}, \mathrm{Ho}, \mathrm{Er}, \mathrm{Yb}, \mathrm{Lu}, \mathrm{Y}, \mathrm{Nb}, \mathrm{Zr}, \mathrm{Th}$, and $\mathrm{U}$.

A close scrutiny of Table 3 reveals that the basalts differ markedly in their $\mathrm{TiO}_{2}$ contents and may be divided into two groups, the averages of which are significantly different. Accordingly, we have discerned lower $\mathrm{Ti}$ and higher $\mathrm{Ti}$ basalts. Once the grouping is made, further distinctions become obvious for other elements. To obtain a suitable basis for comparison, the actual analytical data have been recalculated on a $\mathrm{H}_{2} \mathrm{O}$ and $\mathrm{CO}_{2}$-free basis. The averages for the two groups are listed together with the standard deviation in Table 4. Along with the quite pronounced differences in $\mathrm{TiO}_{2}$, a similar clustering of values occurs for $\mathrm{Al}_{2} \mathrm{O}_{3}, \mathrm{Na}_{2} \mathrm{O}$, and $\mathrm{P}_{2} \mathrm{O}_{5}$. Their respective concentrations versus $\mathrm{TiO}_{2}$ are shown in Figure 5 . The higher Ti group is enriched in $\mathrm{SiO}_{2}, \mathrm{Fe}_{2} \mathrm{O}_{3}, \mathrm{Na}_{2} \mathrm{O}, \mathrm{P}_{2} \mathrm{O}_{5}$, and rare earth element (REE) concentrations, whereas the lower Ti group is enriched in $\mathrm{Al}_{2} \mathrm{O}_{3}, \mathrm{MgO}$, and $\mathrm{CaO}$.

All basalts from Site 373 A show secondary hydration and oxidation (Figure 6). Samples 5-1, 68-75cm and $5-2,35-38 \mathrm{~cm}$, are high in $\mathrm{CaCO}_{3}$.

On an alkali-silica diagram, the Hole $373 \mathrm{~A}$ basalts fall close to the alkaline field boundary or even in the high-alumina basalt field (Figure 7). Most samples are 


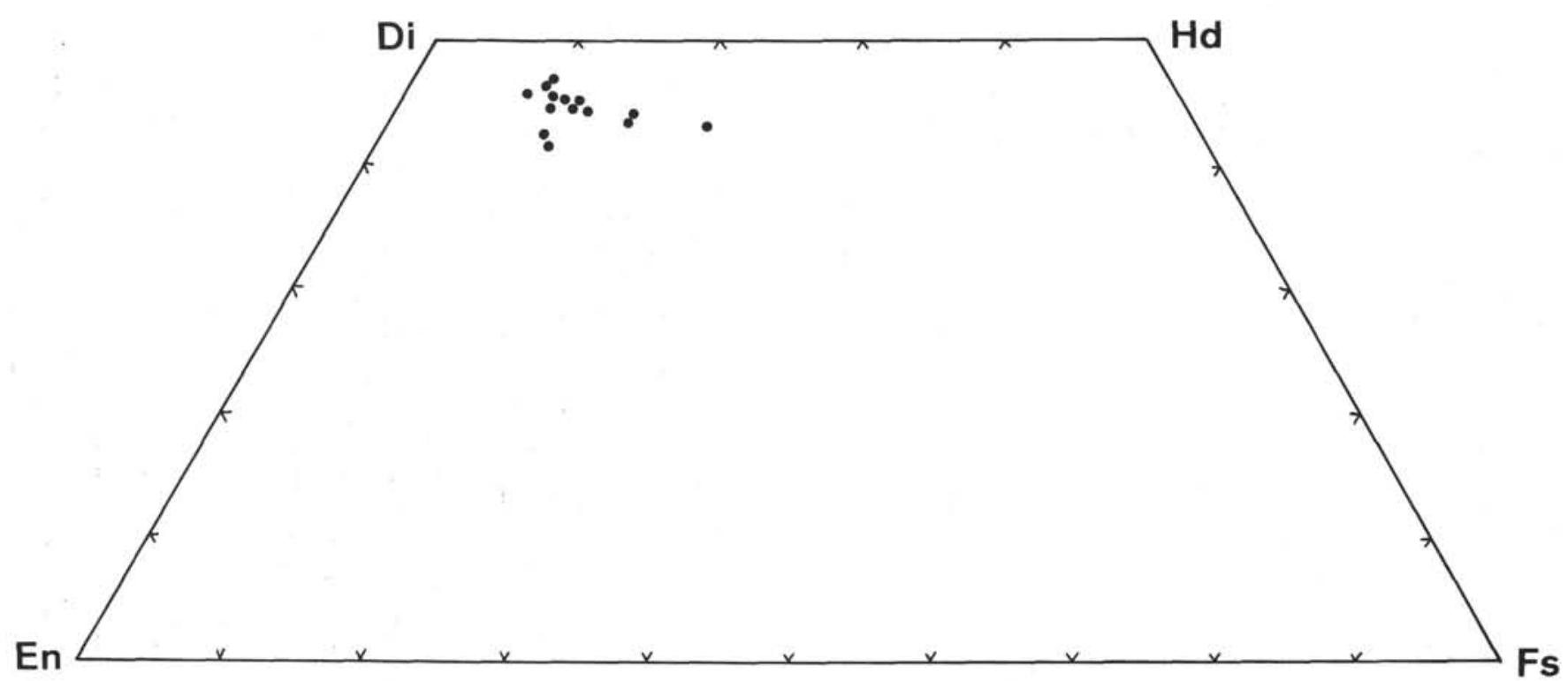

Figure 4. Clinopyroxene plot for Hole 373A basalts.

nepheline-normative (Table 5 and Figure 8) using a corrected iron oxidation of $\mathrm{Fe}_{2} \mathrm{O}_{3} / \mathrm{FeO}=0.15$. This may not reflect the original chemistry. $\mathrm{Na}_{2} \mathrm{O}$ could have been introduced (spilitization). In some of these samples the content of $\mathrm{CaO}$ is $7-8 \%$. This is well below oceanic tholeiite concentrations whose range is exemplified, for example, in the $\mathrm{CaO} / \mathrm{Al}_{2} \mathrm{O}_{3}$ plot of Kay et al. (1970). Miyashiro et al. (1971) reported considerable loss of $\mathrm{CaO}$ with increase of $\mathrm{Na}_{2} \mathrm{O}$ in metabasalts of the Mid-Atlantic Ridge.

On the other hand, the higher $\mathrm{Ti}$ basalts show a consistent enrichment of $\mathrm{SiO}_{2}, \mathrm{Na}_{2} \mathrm{O}, \mathrm{Fe}_{2} \mathrm{O}_{3}, \mathrm{P}_{2} \mathrm{O}_{5}$, and REE.

The data suggest that the two groups represent two initially different basalt types (Figures 7, 9, 10, 11,12, 13, and 14). The higher Ti basalts have higher normative contents of the $\mathrm{Fe}$-Ti-oxides and apatite, and lower contents of olivine (Table 5 and Figure 8). Each type has a different degree of fractionation (Figure 10). The $\mathrm{MgO} / \mathrm{FeO}$ ratio expressed as $\mathrm{Mg}$-numbers (molecular $100 \mathrm{Mg} / \mathrm{Mg}+\mathrm{Fe}^{+2}$ (Green and Ringwood, 1967). The $\mathrm{Fe}^{+2}$, standardized as $\mathrm{Fe}_{2} \mathrm{O}_{3} / \mathrm{FeO}=0.15$, shows a broad range of fractionation with $\mathrm{Mg}$-numbers from 74 to 46 (Table 5). The higher Ti basalts (Mg-numbers: 46-66 and differentiation index: 33-41) could represent a more fractionated part of the magma than the lower Ti basalts. The highest Mg-numbers (65-74 for the lower $\mathrm{Ti}$ basalts) are consistent with a direct partial melting from upper mantle peridotite (Green, 1970). Magma segregation following about $15 \%$ partial melting at about $9 \mathrm{~kb}$ at a depth range of $15-35 \mathrm{~km}$ would yield a high-alumina olivine tholeiite of appropriate composition. The low $\mathrm{TiO}_{2}$, very high $\mathrm{Al}_{2} \mathrm{O}_{3}$ and high normative olivine content (up to $20 \%$ ) may require a refinement of the model with a greater degree of partial melting in a Al-rich source. The lower Ti basalts could be plagioclase-tholeiites of Shido et al. (1971). However, this terminology is not adopted, because the alteration of olivine obscures the crystallization sequence.

\section{TRACE ELEMENTS}

Trace element concentrations are given in Table 3. Chondrite normalized REE patterns are shown in Figure 14.

Concentrations of large-ion-lithophile (LIL) elements (such as $\mathrm{Rb}, \mathrm{Sr}, \mathrm{Ba}, \mathrm{Y}, \mathrm{Zr}, \mathrm{Hf}, \mathrm{U}$ ) are low, indicating that these basalts are similar to abyssal tholeiites. Correlation plots of alteration-resistant element pairs, such as $\mathrm{Zr}-\mathrm{Sr}, \mathrm{Zr}-\mathrm{Nb}$, or $\mathrm{P}_{2} \mathrm{O}_{5}-\mathrm{TiO}_{2}$ (not illustrated), clearly fall in the field of oceanic ridge basalts (Bass et al., 1973; Ridley et al., 1974). Zr-Ti and Y-Ti correlations also fall on the positive correlation line outlined for ocean floor basaltic rocks by Cann (1970). This emphasizes that $\mathrm{Zr}$ and $\mathrm{Y}$ have slightly lower than average concentrations in the Tyrrhenian lower $\mathrm{Ti}$ basalts, and that they are markedly higher in the higher $\mathrm{Ti}$ basalts. $\mathrm{La}$ and $\mathrm{Hf}$ also are different in the two basalt types previously distinguished (Figure 13).

$\mathrm{Rb}, \mathrm{Sr}, \mathrm{Ba}, \mathrm{Pb}, \mathrm{U}$, and $\mathrm{Th}$ have concentrations slightly but consistently above those found in ocean floor basalts (Engel et al., 1965; Kay et al., 1970; Hart, 1971; Church and Tatsumoto, 1975).

Relative to chondrites, the shape of the rare earth patterns and the Eu anomalies (Figure 14) are interesting. All samples have a similar general REE pattern. They are light $\mathrm{REE}$ enriched with $(\mathrm{La} / \mathrm{Sm})_{\text {e.f. }}$ of about 1.5. The enrichment of the heavy REE shows differences which coincide with the grouping into the lower $\mathrm{Ti}$ and higher $\mathrm{Ti}$ groups of basalts (Table 4 and Figures 7-13). This high (Ls/Sm) e.f. is a distinctive feature of our REE patterns and is at variance with abyssal tholeiites which typically have $(\mathrm{La} / \mathrm{Sm})_{\text {e.f. }}<1$ (Schilling, 1971). 
TABLE 3

Chemical Composition of the Leg 42A Hole 373A Basalts, (n.d. = not determined)

\begin{tabular}{|c|c|c|c|c|c|c|c|c|c|c|c|c|c|c|c|c|c|c|c|c|}
\hline & (1) & (2) & (3) & (4) & (5) . & (6) & (7) & (8) & (9) & (10) & (11) & (12) & (13) & (14) & (15) & (16) & (17) & (18) & (19) & (20) \\
\hline $\begin{array}{l}\text { DSDP } \\
\text { Leg 42A-373A }\end{array}$ & $\begin{array}{c}5-1 \\
68-71\end{array}$ & $\begin{array}{c}5-1 \\
90-93\end{array}$ & $\begin{array}{c}5-2 \\
35-38\end{array}$ & $\begin{array}{c}5-2 \\
53.5-57\end{array}$ & $\begin{array}{c}5-3 \\
74-75.5\end{array}$ & $\begin{array}{c}5-3 \\
129-131\end{array}$ & $\begin{array}{c}\text { 6-1 } \\
96-100\end{array}$ & $6 \mathrm{cc}$ & $\begin{array}{c}7-1 \\
139-144\end{array}$ & $\begin{array}{c}7-2 \\
136-140\end{array}$ & $\begin{array}{c}7-3 \\
111-1125\end{array}$ & $\begin{array}{c}7-4 \\
19-23\end{array}$ & $\begin{array}{c}7-4 \\
135-140\end{array}$ & $\begin{array}{c}7-5 \\
10-14\end{array}$ & $\begin{array}{c}10-1 \\
123-125\end{array}$ & $\begin{array}{c}11-1 \\
114-117\end{array}$ & $\begin{array}{c}11-1 \\
130-135\end{array}$ & $12 \mathrm{cc}$ & $\begin{array}{l}\text { OB } 2 \\
\text { Outer }\end{array}$ & $\underset{\text { barrel }}{\text { Ocb }}$ \\
\hline $\begin{array}{l}\mathrm{SiO}_{2} \\
\mathrm{TiO}_{2} \\
\mathrm{Al}_{2} \mathrm{O}_{3} \\
\mathrm{Fe}_{2} \mathrm{O}_{3} \\
\mathrm{FeO} \\
\mathrm{MnO} \\
\mathrm{MgO} \\
\mathrm{CaO} \\
\mathrm{Na}_{2} \mathrm{O} \\
\mathrm{K}_{2} \mathrm{O} \\
\mathrm{P}_{2} \mathrm{O}_{5} \\
\mathrm{H}_{2} \mathrm{O} \\
\mathrm{CO}_{2}\end{array}$ & $\begin{array}{c}40.9 \\
1.12 \\
17.8 \\
5.12 \\
2.78 \\
0.23 \\
.7 .03 \\
14.1 \\
3.02 \\
0.32 \\
0.20 \\
3.71 \\
4.42\end{array}$ & $\begin{array}{c}49.2 \\
1.08 \\
18.7 \\
4.48 \\
2.80 \\
0.11 \\
7.03 \\
10.5 \\
2.93 \\
0.30 \\
0.17 \\
2.49 \\
<0.1\end{array}$ & $\begin{array}{c}41.5 \\
1.07 \\
17.9 \\
5.40 \\
2.18 \\
0.17 \\
7.9 \\
12.9 \\
2.43 \\
0.28 \\
0.16 \\
4.80 \\
4.12\end{array}$ & $\begin{array}{c}47.1 \\
1.03 \\
18.9 \\
5.29 \\
2.30 \\
0.11 \\
7.60 \\
11.4 \\
2.97 \\
0.32 \\
0.12 \\
2.40 \\
0.1\end{array}$ & $\begin{array}{c}48.1 \\
1.55 \\
17.4 \\
6.89 \\
2.95 \\
0.19 \\
4.83 \\
10.4 \\
3.84 \\
0.29 \\
0.26 \\
1.88 \\
0.1\end{array}$ & $\begin{array}{c}48.4 \\
1.71 \\
16.1 \\
6.61 \\
3.38 \\
0.19 \\
5.72 \\
10.2 \\
4.22 \\
0.43 \\
0.23 \\
2.38 \\
<0.1\end{array}$ & $\begin{array}{c}51.1 \\
1.75 \\
17.1 \\
7.16 \\
2.98 \\
0.17 \\
4.02 \\
9.5 \\
4.65 \\
0.38 \\
0.25 \\
0.92 \\
<0.1\end{array}$ & $\begin{array}{c}49.2 \\
1.41 \\
16.1 \\
5.63 \\
3.84 \\
0.16 \\
7.54 \\
10.0 \\
3.61 \\
0.32 \\
0.19 \\
1.97 \\
<0.1\end{array}$ & $\begin{array}{c}48.0 \\
0.92 \\
18.3 \\
5.19 \\
2.65 \\
0.10 \\
7.66 \\
10.3 \\
3.16 \\
0.33 \\
0.12 \\
3.39 \\
<0.1\end{array}$ & $\begin{array}{c}47.8 \\
0.84 \\
20.2 \\
4.82 \\
4.95 \\
0.13 \\
7.56 \\
11.17 \\
2.61 \\
0.27 \\
0.10 \\
2.51 \\
0.1\end{array}$ & $\begin{array}{c}47.7 \\
1.02 \\
18.2 \\
5.28 \\
2.81 \\
0.16 \\
8.35 \\
11.8 \\
2.73 \\
0.21 \\
0.13 \\
2.41 \\
<0.1\end{array}$ & $\begin{array}{c}44.5 \\
0.84 \\
20.3 \\
5.06 \\
2.50 \\
0.15 \\
8.65 \\
10.8 \\
2.83 \\
0.24 \\
0.12 \\
3.22 \\
<0.1\end{array}$ & $\begin{array}{c}43.2 \\
0.91 \\
18.0 \\
5.54 \\
2.51 \\
0.17 \\
10.3 \\
11.1 \\
2.53 \\
0.23 \\
0.11 \\
4.44 \\
1.5\end{array}$ & $\begin{array}{c}44.2 \\
1.09 \\
18.2 \\
5.9 \\
3.30 \\
0.27 \\
9.58 \\
10.3 \\
2.27 \\
0.25 \\
0.14 \\
4.58 \\
0.8\end{array}$ & $\begin{array}{c}49.2 \\
1.11 \\
18.1 \\
4.24 \\
2.47 \\
0.12 \\
8.76 \\
9.24 \\
3.24 \\
0.27 \\
0.14 \\
3.00 \\
<0.1\end{array}$ & $\begin{array}{c}51.0 \\
1.68 \\
16.5 \\
5.65 \\
4.29 \\
0.17 \\
7.05 \\
7.57 \\
4.13 \\
0.27 \\
0.24 \\
2.27 \\
<0.1\end{array}$ & $\begin{array}{c}50.5 \\
1.57 \\
16.9 \\
4.71 \\
4.50 \\
0.16 \\
7.95 \\
7.3 \\
4.28 \\
0.28 \\
0.22 \\
2.14 \\
<0.1\end{array}$ & $\begin{array}{c}50.0 \\
1.56 \\
16.4 \\
4.81 \\
4.03 \\
0.16 \\
8.13 \\
7.0 \\
4.40 \\
0.32 \\
0.22 \\
2.31 \\
<0.1\end{array}$ & $\begin{array}{c}50.1 \\
1.72 \\
16.8 \\
4.98 \\
4.23 \\
0.18 \\
7.21 \\
7.70 \\
4.18 \\
0.32 \\
0.26 \\
2.12 \\
<0.1\end{array}$ & $\begin{array}{c}50.6 \\
1.86 \\
16.3 \\
5.20 \\
4.33 \\
0.16 \\
7.01 \\
7.23 \\
4.44 \\
0.37 \\
0.26 \\
2.56 \\
<0.1\end{array}$ \\
\hline Total & 100.75 & 99.8 & 100.8 & 99.54 & 98.63 & 99.57 & 99.98 & 100.02 & 100.12 & 99.8 & 100.89 & 99.21 & 100.54 & 100.89 & 99.89 & 100.22 & 100.51 & 99.34 & 99.80 & 100.32 \\
\hline $\begin{array}{l}\mathrm{Li} \\
\mathrm{Rb} \\
\mathrm{Sr} \\
\mathrm{Ba} \\
\mathrm{Pb} \\
\mathrm{Cu} \\
\mathrm{Co}\end{array}$ & $\begin{array}{c}10 \\
<1 \\
200 \\
\text { n.d. } \\
\text { n.d. } \\
68 \\
29\end{array}$ & $\begin{array}{c}29 \\
<1 \\
250 \\
\text { n.d. } \\
\text { n.d. } \\
76 \\
32\end{array}$ & $\begin{array}{c}33 \\
<1 \\
225 \\
\text { n.d. } \\
\text { n.d. } \\
86 \\
33\end{array}$ & $\begin{array}{c}32 \\
<1 \\
215 \\
\text { n.d. } \\
\text { n.d. } \\
76 \\
29\end{array}$ & $\begin{array}{c}18 \\
<1 \\
230 \\
\text { n.d. } \\
\text { n.d. } \\
98 \\
37\end{array}$ & $\begin{array}{c}27 \\
<1 \\
220 \\
\text { n.d. } \\
\text { n.d. } \\
72 \\
31\end{array}$ & $\begin{array}{c}9 \\
<1 \\
160 \\
\text { n.d. } \\
\text { n.d. } \\
26 \\
32\end{array}$ & $\begin{array}{c}29 \\
<1 \\
205 \\
\text { n.d. } \\
\text { n.d. } \\
84 \\
35\end{array}$ & $\begin{array}{c}29 \\
<2.5 \\
232 \\
58.5 \\
2.2 \\
61 \\
30\end{array}$ & $\begin{array}{c}27 \\
<1 \\
215 \\
\text { n.d. } \\
\text { n.d. } \\
66 \\
39\end{array}$ & $\begin{array}{c}29 \\
<1 \\
225 \\
\text { n.d. } \\
\text { n.d. } \\
69 \\
33\end{array}$ & $\begin{array}{c}34 \\
3 \\
237 \\
\text { n.d. } \\
1.5 \\
67 \\
34\end{array}$ & $\begin{array}{c}50 \\
2 \\
215 \\
\text { n.d. } \\
1.5 \\
84 \\
31\end{array}$ & $\begin{array}{c}46 \\
<1 \\
235 \\
\text { n.d. } \\
\text { n.d. } \\
150 \\
39\end{array}$ & $\begin{array}{c}34 \\
<1 \\
210 \\
\text { n.d. } \\
\text { n.d. } \\
105 \\
35\end{array}$ & $\begin{array}{c}11 \\
<1 \\
180 \\
\text { n.d. } \\
\text { n.d. } \\
84 \\
32\end{array}$ & $\begin{array}{c}17 \\
<1 \\
205 \\
58 \\
2.4 \\
70 \\
32\end{array}$ & $\begin{array}{c}11 \\
<1 \\
155 \\
\text { n.d. } \\
\text { n.d. } \\
68 \\
30\end{array}$ & $\begin{array}{c}8 \\
<1 \\
197 \\
\text { n.d. } \\
\text { n.d. } \\
76 \\
31\end{array}$ & $\begin{array}{c}15 \\
<1 \\
230 \\
\text { n.d. } \\
\text { n.d. } \\
72 \\
31\end{array}$ \\
\hline $\mathrm{Cr}$ & 201 & 199 & 206 & 207 & 190 & 199 & 169 & 207 & 227 & 285 & 255 & 253 & 210 & 210 & 221 & 138 & 133 & 142 & 115 & 100 \\
\hline $\begin{array}{l}\text { Sc } \\
\text { Y }\end{array}$ & $\begin{array}{l}34 \\
\text { n.d. }\end{array}$ & $\begin{array}{l}32 \\
28\end{array}$ & $\begin{array}{l}31 \\
28\end{array}$ & $\begin{array}{l}29 \\
\text { n.d. }\end{array}$ & $\begin{array}{l}39 \\
\text { n.d. }\end{array}$ & $\begin{array}{l}35 \\
37\end{array}$ & $\begin{array}{l}36 \\
\text { n.d. }\end{array}$ & $\begin{array}{l}43 \\
28\end{array}$ & $\begin{array}{l}29 \\
23\end{array}$ & $\begin{array}{l}32 \\
\text { n.d. }\end{array}$ & $\begin{array}{l}33 \\
22\end{array}$ & $\begin{array}{l}30 \\
18\end{array}$ & $\begin{array}{l}33 \\
21\end{array}$ & $\begin{array}{l}34 \\
26\end{array}$ & $\begin{array}{l}35 \\
28\end{array}$ & $\begin{array}{l}32 \\
40\end{array}$ & $\begin{array}{l}34 \\
39\end{array}$ & $\begin{array}{l}33 \\
\text { n.d. }\end{array}$ & $\begin{array}{l}32 \\
43\end{array}$ & $\begin{array}{l}29 \\
36\end{array}$ \\
\hline $\mathrm{La}$ & 5.8 & 5.6 & 5.2 & 6.2 & 7.3 & 9.3 & 9.1 & 6.1 & 6.3 & 3.7 & 4.7 & 6.1 & 5.3 & 4.5 & 5.2 & 9.3 & 9.1 & 9.2 & 8.7 & 10.0 \\
\hline $\mathrm{Ce}$ & 14.0 & 10.9 & 11.5 & 11.9 & 16.0 & 20.1 & 22.9 & 15.1 & 15.0 & 8.9 & 11.8 & 13.0 & 12.0 & 11.1 & 12.2 & 20.0 & 20.2 & 20.3 & 18.9 & 20.5 \\
\hline $\mathrm{Sm}$ & 2.6 & 1.9 & 2.0 & 2. & 3. & 3. & 3. & 3.2 & 1.9 & 1.8 & 2.1 & 2. & 3.4 & 2. & 2.2 & 2.9 & 3. & 3.7 & 3.8 & 4.2 \\
\hline $\mathrm{Eu}$ & 0.86 & 1.04 & 0.82 & 1.10 & 1.44 & 1.43 & 1.37 & 1.25 & 0.90 & 0.84 & 0.89 & 0.92 & 0.71 & 0.85 & 0.98 & 1.43 & 1.24 & 1.34 & 1.30 & 1.57 \\
\hline $\mathrm{Tb}$ & 1.1 & $<1$ & $<1$ & $<1$ & 1.5 & 1.5 & 1.2 & 1.0 & 0.63 & $<1$ & $<1$ & $<1$ & $<1$ & 1.1 & $<1$ & 1.6 & $<1$ & $<1$ & 1.2 & $<1$ \\
\hline Dy & 3. & 3.0 & 3.4 & 3.2 & & & & 4 & $<1$ & 2.6 & 3.2 & 2.8 & 3.2 & 3 & 3.5 & & 5.6 & 5.2 & 5 & 5.3 \\
\hline $\mathrm{Yb}$ & 2.2 & 1 & & & & 3.5 & & 2.9 & 2.2 & 1.5 & 1.8 & $<1$ & 1.1 & 2.2 & 2.3 & 3.0 & 3.4 & 3.5 & 2.8 & 2.5 \\
\hline Lu & 0.27 & 0.26 & 0.28 & 0.27 & 0.40 & 0.43 & 0.37 & 0.38 & 0.29 & 0.26 & 0.31 & 0.28 & 0.25 & 0.30 & 0.30 & 0.46 & 0.49 & 0.42 & 0.46 & 0.50 \\
\hline $\mathrm{Z}_{\mathrm{r}}$ & n.d. & 81 & 82 & n.d. & n.d. & 142 & n.d. & 107 & 77 & n.d. & 69 & 61 & 54 & 79 & 90 & 156 & 140 & n.d. & 165 & 153 \\
\hline $\mathrm{Hf}$ & 2.2 & $<1$ & 2.0 & 0.9 & 2.7 & 2.7 & & & 1.6 & 1.9 & 2.0 & 1.5 & 1.2 & 2.0 & 1.5 & 3.3 & 3.2 & 3.3 & 2.4 & 3.1 \\
\hline $\mathrm{Nb}$ & n.d. & n.d. & n.d. & n.d. & n.d. & n.d. & n.d. & n.d. & 3.1 & n.d. & n.d. & n.d. & n.d. & n.d. & n.d. & n.d. & 6.5 & n.d. & n.d. & n.d. \\
\hline $\mathrm{Ta}$ & $<1$ & $<1$ & $<1$ & $<1$ & 0.85 & $<1$ & $<1$ & 0.77 & $<.1$ & $<.1$ & $<1$ & $<1$ & $<1$ & 0.95 & $<1$ & $<1$ & 1.08 & $<1$ & $<1$ & $<1$ \\
\hline Th & $<.3$ & $<.3$ & $<.3$ & $<.3$ & $<.3$ & $<.3$ & $<.3$ & $<.3$ & 0.4 & $<.3$ & $<.3$ & $<.3$ & $<.3$ & $<.3$ & $<.3$ & $<.3$ & 0.63 & $<.3$ & $<.3$ & $<.3$ \\
\hline U & $<.2$ & $<.2$ & $<.2$ & $<.2$ & $<.2$ & $<.2$ & $<.2$ & $<.2$ & 0.24 & $<.2$ & $<.2$ & 0.11 & 0.21 & $<.2$ & $<.2$ & $<.2$ & 0.8 & $<.2$ & $<.2$ & $<.2$ \\
\hline
\end{tabular}


TABLE 4

Chemical and Petrographical Characteristics of the Two Basalt Types of Hole 373A on a (dry) Volatile Free Basis

\begin{tabular}{lcc} 
& $\begin{array}{c}\text { Lower Ti } \\
\text { Group } \\
(11 \text { samples }) \\
\overline{\mathrm{X}} \pm 1 \mathrm{~S}\end{array}$ & $\begin{array}{c}\text { Higher Ti } \\
\text { Group } \\
(9 \text { samples })\end{array}$ \\
\hline$\overline{\mathrm{X}} \pm 1 \mathrm{~S}$
\end{tabular}

The positive Eu anomaly is obviously linked to samples with high $\mathrm{Al}_{2} \mathrm{O}_{3}$ contents, indicating that plagioclase is the Eu-bearing phase.

\section{PETROGENETIC DISCUSSION}

Drilling on Leg 42A encountered, for the first time, abyssal basalts in the young Mediterranean deep-sea basins. Two significantly different and interlayered types of basalts were recovered from Hole 373A; lower Ti basalts with affinities to high-alumina abyssal tholeiites, and higher Ti basalts.

Alteration of these basalts is widespread and involves chemical migrations. It is most evident in the $\mathrm{CaCO}_{3}$ bearing samples and in apparently $\mathrm{Na}$-enriched and $\mathrm{Ca}$-depleted samples (spilitization). The ocean floor metamorphic processes responsible are probably related to circulation of hydrothermal solutions in areas of higher heat flow; they merit further study. At present, we concentrate primarily upon the recognition of original magma types.

According to Green and Ringwood (1967, 1969), 01-normative, high-alumina tholeiite (the Site 373A low-Ti basalts) is generated at a relatively shallow mantle depth (about $9 \mathrm{~kb}$ or a model depth of 15-35 $\mathrm{km}$ ) and by high degree of partial melting (25\%-30\%). The origin of basalt within these limitations is thought to be related to upwelling or intrusion of partly molten mantle material from the low-velocity zone into shallower levels of a narrow axial zone at spreading centers. High heat flow is related to the rising, hot mantle material. Basalts of the lower $\mathrm{Ti}$ group with highest $\mathrm{Mg}$ numbers can be directly related to this model and do not require large-scale fractionation processes to account for their composition. Plagioclase accumulation can explain the extremely high $\mathrm{AlO}_{3}$ enrichment and a possibly existing Eu anomaly related to high $\mathrm{Sr}$ contents.

Variations in LIL and other incompatible elements between abyssal basalts from different regions may
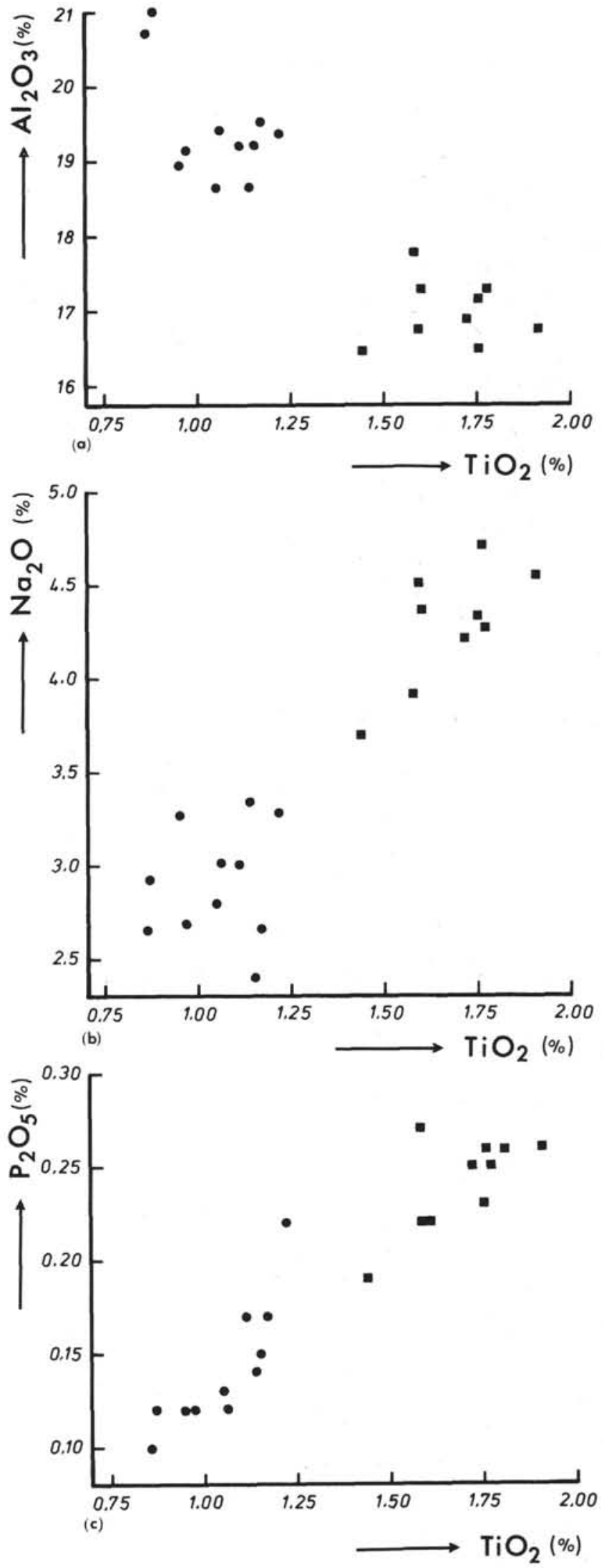

Figure 5. Variation diagrams for Hole $373 \mathrm{~A}$ basalts. (a) $\mathrm{Al}_{2} \mathrm{O}_{3} / \mathrm{TiO}_{2}$, (b) $\mathrm{Na}_{2} \mathrm{O} / \mathrm{TiO}_{2}$; (c) $\mathrm{P}_{2} \mathrm{O}_{5} / \mathrm{TiO}_{2}$. Solid circles $=$ low $-\mathrm{Ti}$ basalts; solid squares $=$ high-Ti basalts. For location see Figure 2. 


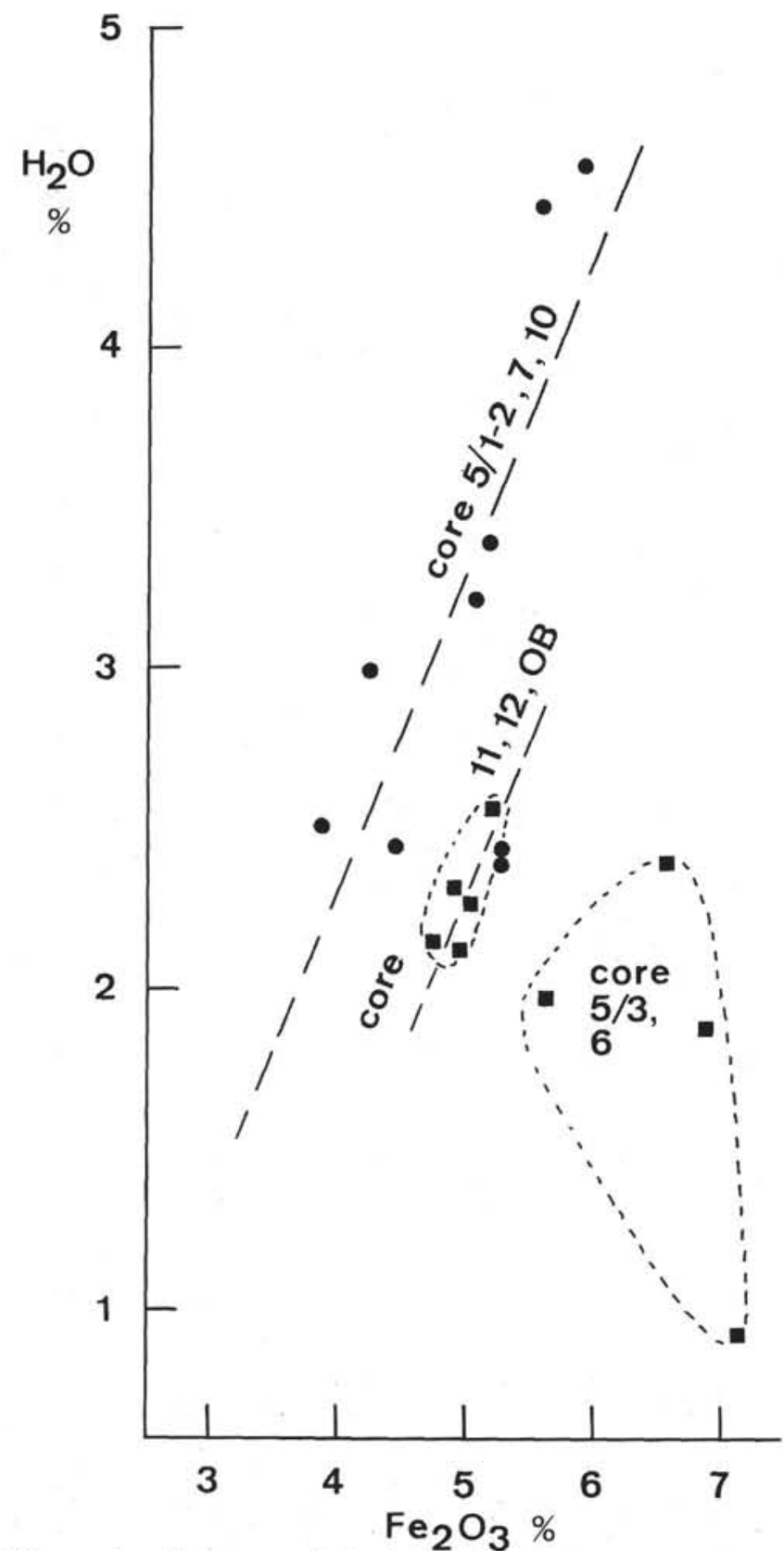

Figure 6. Increase of $\mathrm{Fe}_{2} \mathrm{O}_{3}$ and $\mathrm{H}_{2} \mathrm{O}$ within the $373 \mathrm{~A}$ basalts indicating the degree of weathering and metamorphism. Solid circles $=$ Ti basalts, solid squares $=$ high Ti-basalts.

reflect different concentrations in the source rocks. The source of the most unfractionated Tyrrhenian basalts yields lower $\mathrm{Ti}, \mathrm{Y}, \mathrm{Zr}$ and is enriched in light REE, Ba, $\mathrm{Rb}, \mathrm{Sr}, \mathrm{Th}, \mathrm{U}, \mathrm{Pb}$, compared with oceanic basalts. Kay et al. (1970) showed that extremely low LIL element concentrations along the Mid-Atlantic Ridge resulted from depletion during previous partial melting episodes. The depletion under the Tyrrhenian Basin is less pronounced.

A postulated vertical zoning of the upper mantle, reflected in the enrichment of LIL elements in the upper levels of the low velocity zone (Green, 1970), can also lead to increased LIL element concentrations

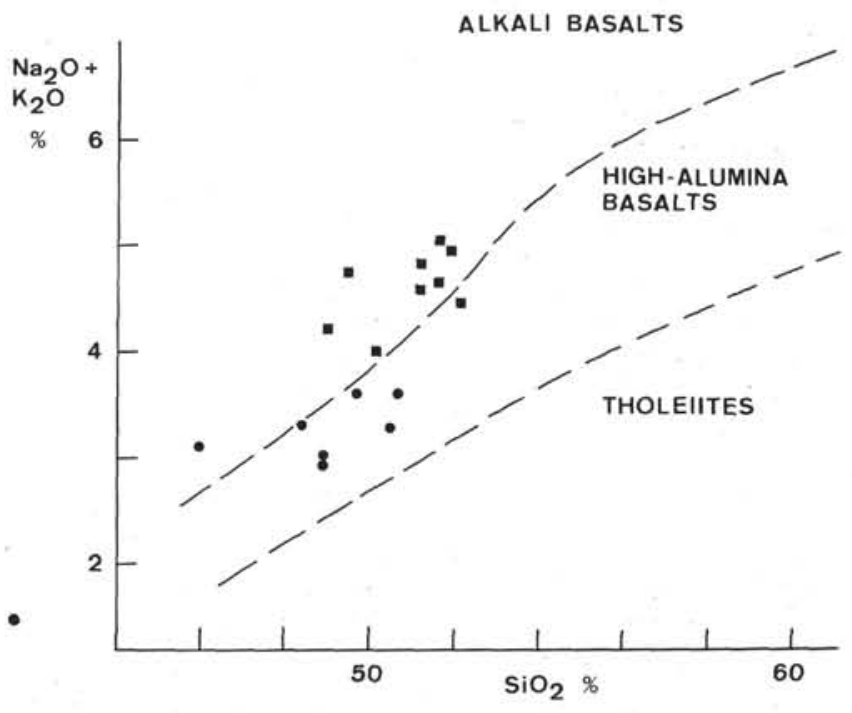

Figure 7. Alkali $/ \mathrm{SiO}_{2}$ plot on water-free basis $($ solid circles $=$ low-Ti basalts, solid squares $=$ high-Ti basalts).

in partial melts. If the upper mantle below the Tyrrhenian Sea has a structure similar to that beneath the Balearic Basin (Berry and Knopoff, 1967), then an ultra-low velocity channel upwarp to only $50 \mathrm{~km}$ below the central basin could well provide higher LIL elements even in tholeiites representing high degrees of partial melting.

The Hole 373A higher $\mathrm{Ti}$ basalts could have originated as Qz-normative basalts (containing lower $\mathrm{Na}_{2} \mathrm{O}$ contents, averaging approximately $2.8 \mathrm{wt} \%$ ). Such original rocks apparently were more fractionated than the lower $\mathrm{Ti}$ basalts. No simple fractionation line relates the two groups. To account for the higher $\mathrm{Ti}$ basalts, we envision a complex origin involving a lesser degree of partial melting (to explain higher $\mathrm{Ti}, \mathrm{Hf}, \mathrm{Zr}$,

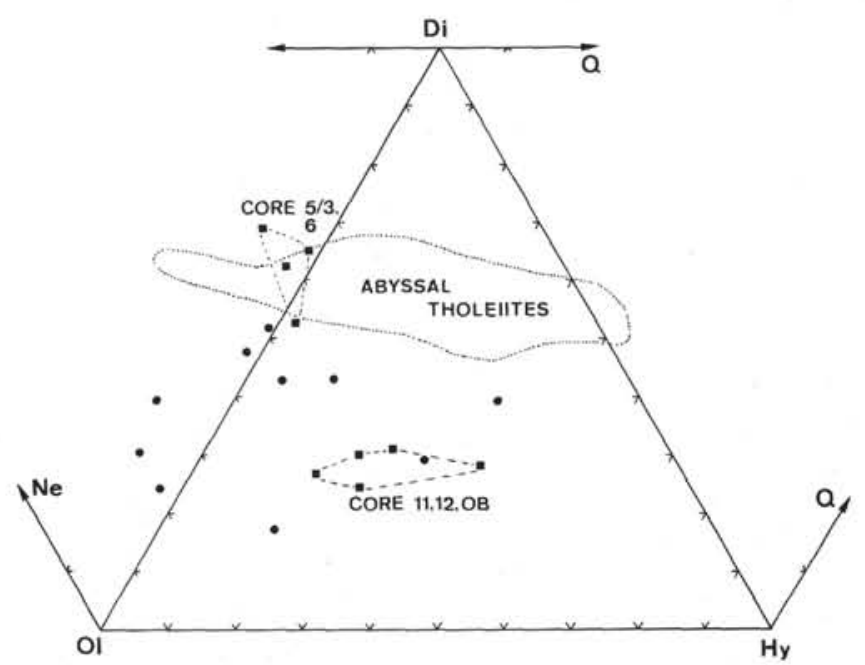

Figure 8. Normative compositions of the Hole $373 \mathrm{~A}$ basalts plotted in the system Ne-01-Di-Hy-Q. The encircled area represents the range of abyssal tholeiites from oceanic ridges. Solid circles $=l o w-T i$ basalts, solid squares $=$ high - Ti basalts. 
TABLE 5

CIPW Norm and Mineralogical Criteria

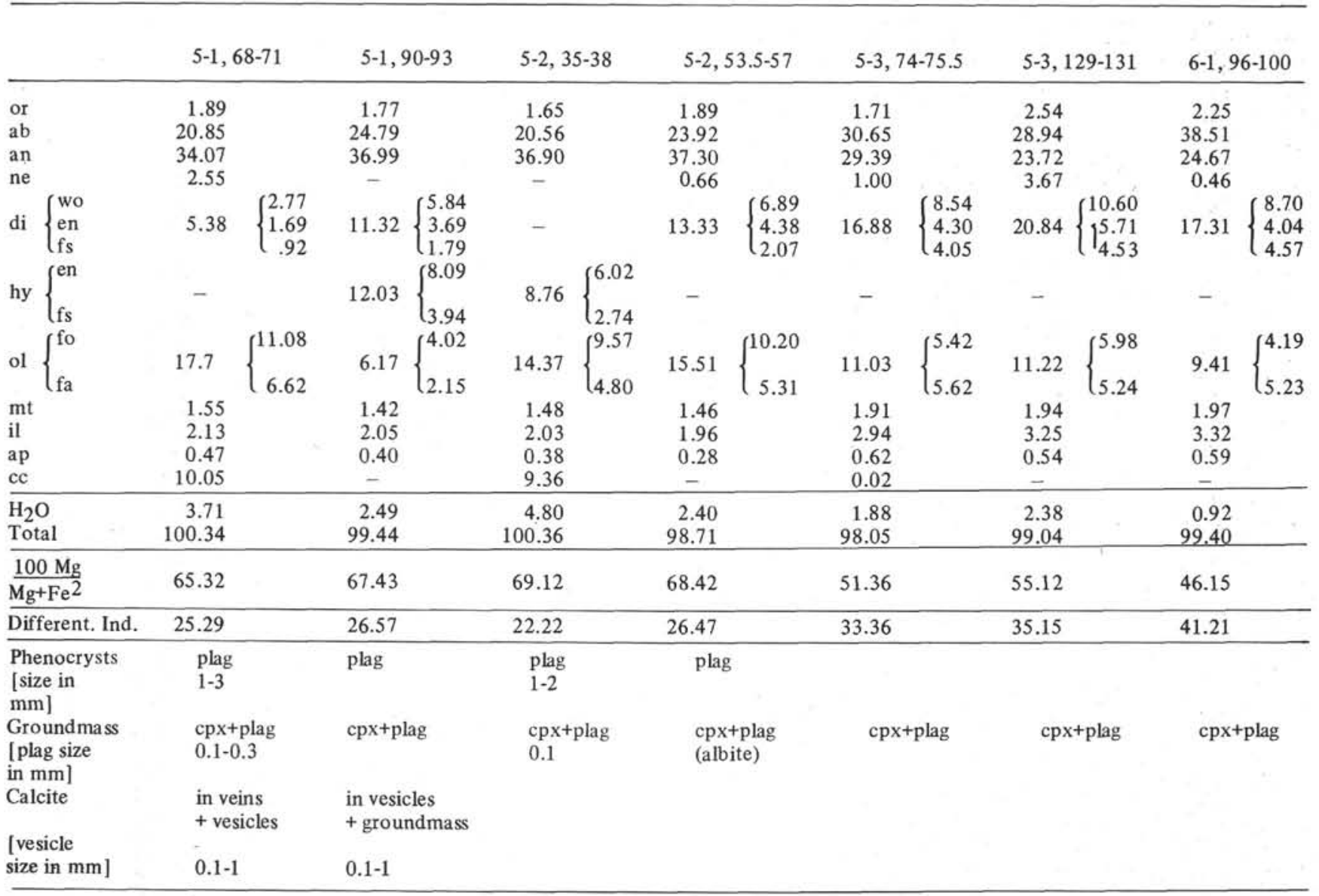

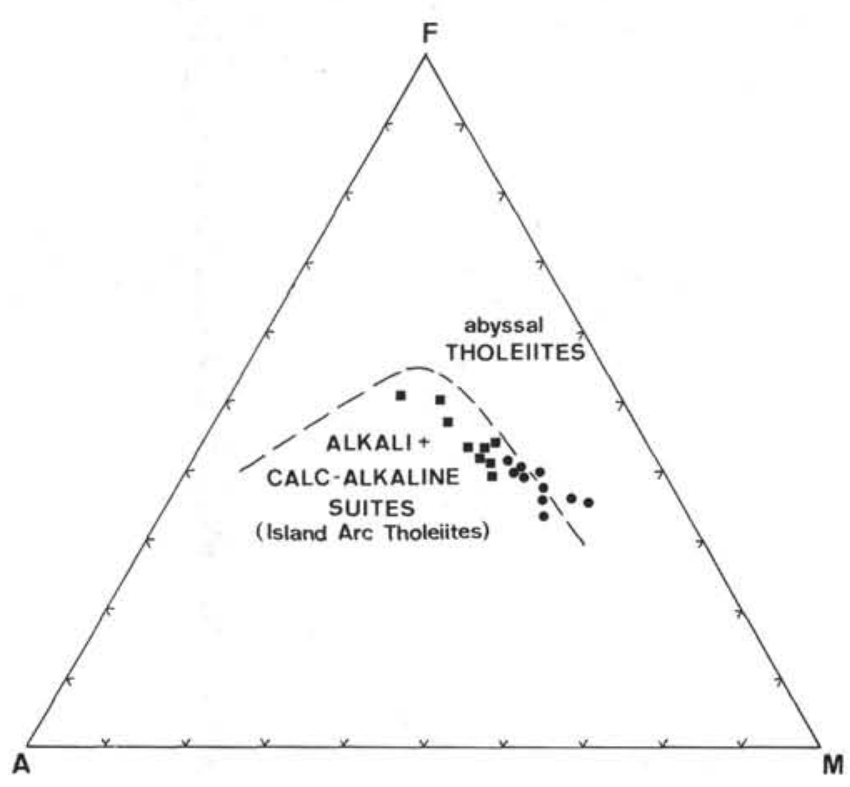

Figure 9. $\mathrm{AFM}$ diagram $\left(\mathrm{Na}_{2} \mathrm{O}+\mathrm{K}_{2} \mathrm{O}\right) / \mathrm{FeO}_{\text {Total }} \mathrm{MgO}$ showing the distribution of 20 analyses of the Hole $373 \mathrm{~A}$ basalts. Solid circles $=$ low-Ti basalts; solid squares $=$ high-Ti basalts.
Y, REE), extensive olivine-pyroxene fractionation, and probably a lack of plagioclase accumulation. But this cannot be further quantified because of the extensive alteration of this group. In conclusion, the trace elements of the Tyrrhenian basalts collected during Leg $42 \mathrm{~A}$ show low LIL element concentrations similar to abyssal tholeiites. Within this level some elements (K, $\mathrm{Rb}, \mathrm{Sr}, \mathrm{Ba}, \mathrm{Pb}, \mathrm{U}$, light REE) have concentrations at the upper limit of the abyssall range, and higher concentrations of these elements in the source rocks. Major element abundances are also similar to those at of abyssal tholeiites (Kay et al., 1970; Cann, 1971; Hart, 1971) and to marginal basin basalts (Hart et al., 1972; Ridley et al., 1974; Hawkins, 1976).

\section{ACKNOWLEDGMENTS}

The authors are indebted to P. Beasly for carrying out the $\mathrm{XRF}$ analysis of some trace elements; N. Ware for instruction in the use of the microprobe; S. R. Taylor and P. Muir for running two cross check samples at the spark source mass spectrometer; and to K. J. Hsü, F. Fabricius and D. Bernoulli for providing the core samples. The samples were irradiated in the Karlsruhe reactor FR2, and were analyzed by U. Kramer with equipment supplied by the Ministry of Research and Technology (FRG). W. G. Ernst read the draft and offered helpful criticism. 
TABLE 5 - Continued

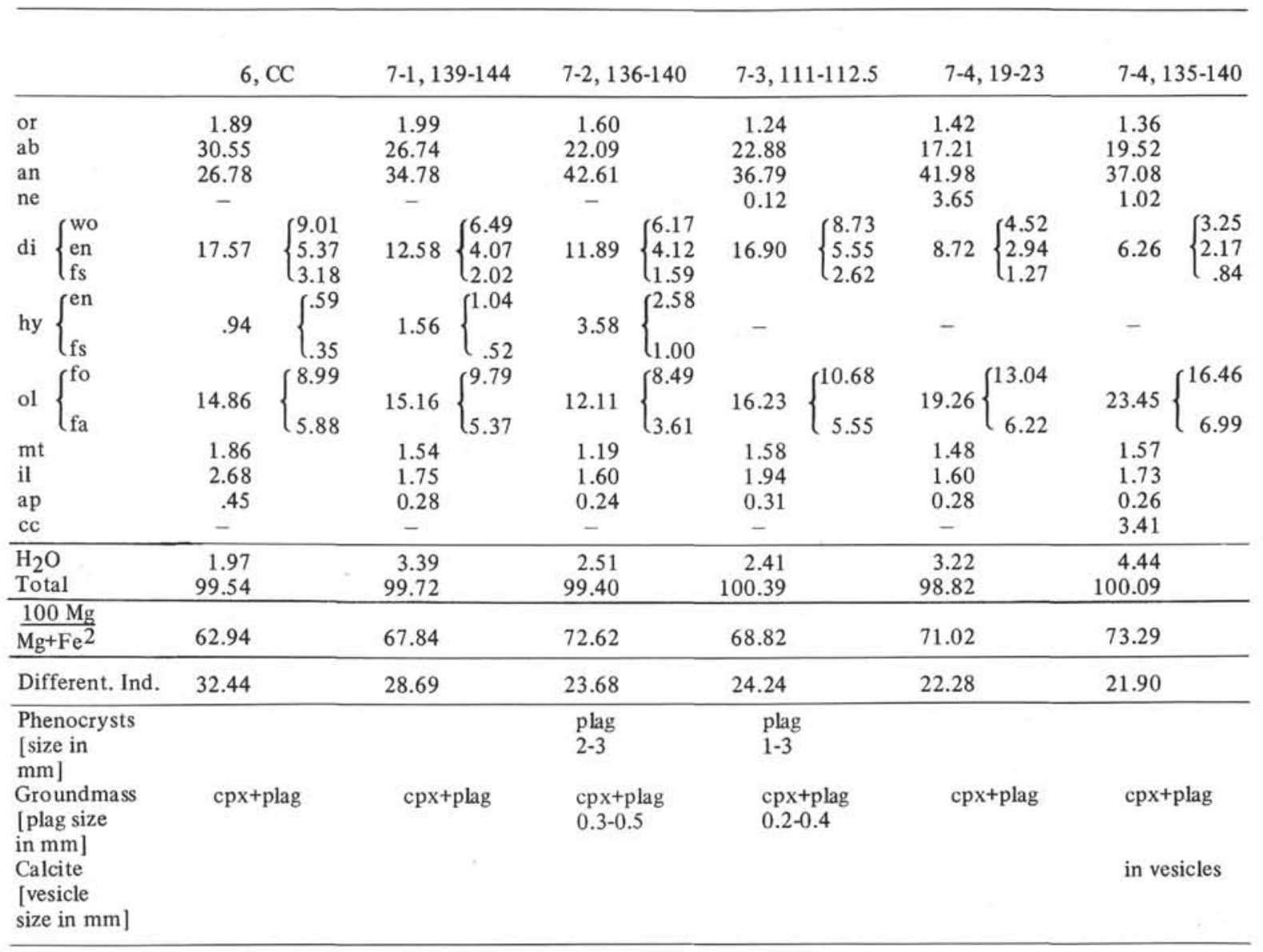

\section{REFERENCES}

Alvarez, W., Cocozza, T. and Wezel, F. C., 1974. Fragmentation of the Alpine orogenic belt by microplate dispersial: Nature, 248, 309-314.

Barberi, F., Bizouard, H., Capaldi, G., Ferrara, G., Gasparini, P., Innocenti F., Lambert, B., and Treuil, M., 1977. Age and nature of basalts from the Tyrrhenian Abyssal Plain; this volume.

Barberi, F., Gasparini, P., Innocenti, F., and Villari, L., 1973. Volcanism if the Southern Tyrrhenian Sea and its Geodynamic implications: J. Geophys. Res., 78, 5221-5232.

Barberi, F ., Innocenti, F., Ferrara, G., Keller, J., Villari, L., 1974. Evolution of Eolian arc volcanism: Earth Planet. Sci. Lett., 21, p. 269-276.

Bass, M. N., Moberly, R., Rhodes, J. M., Shih, C., and Church, S. E., 1973. Volcanic rocks cored in the Central Pacific, Leg 17 DSDP: Am. Geophys. Union Trans., 54, p. 991-995.

Berry, M. J. and Knopoff L., 1967. Structure of the upper mantle under the Western Mediterranean basin: J. Geophys. Res., 72 , p. $3613-3626$.

Boccaletti, M. and Guazzone, G., 1972. Gli archi appeninici, il Mar Ligure ed il Terreno nel guadro della tettonica dei bacini marginali retroareo: Mem. Soc. Geol. It. 11, p. 201216.

Cann, J. R., 1969. Spilites from the Carlsberg Ridge, Indian Ocean: J. Petrol., v. 10, p. 1-19. 1970. Rb, $\mathrm{Sr}, \mathrm{Y}, \mathrm{Zr}$, and $\mathrm{Nb}$ in some ocean floor basaltic rocks: Earth Planet. Sci. Lett., v. 10, p. 7-11.
1971. Major element variations in ocean-floor basalts: Phil. Trans. Roy. Soc. London, Sec. A, v. 268, p. 495-505.

Church, S. E. and Tatsumoto M., 1975. Lead isotopic relations in oceanic ridge basalts from the Juan de Fuca Gorda Ridge area, NE Pacific Ocean: Contrib. Mineral. Petrol.,v. 53, p. $253-279$.

Del Monte, M., 1972. Il vulcanesimo del Mar Tirreno: Nota preliminare sui vulcani Marsili e Palinuro: Giorn. Geol., v. 38, p. 231-252.

Dewey, J. F., Pitman, W. C., Ryan, W. B. F., and Bonin, J., 1973. Plate tectonics and the evolution of the Alpine system: Geol. Soc. Am. Bull., v. 84, p. 3137-3180.

Engel, A. E. J., Engel, C. G., and Havens, R. G., 1965. Chemical characteristics of oceanic basalts and the upper mantle: Geol. Soc. Am. Bull.,v. 76, p. 719-754.

Green, D. H., 1970. The origin of basaltic and nepholinitic magmas: Leichester Lit. Phils. Soc. Trans., v. 64, p. 28-54.

Green, D. H. and Ringwood,, A. E., 1967. The genesis of basaltic magmas: Contrib. Mineral. Petrol., v. 15, p. 103190.

1969. The origin of basaltic magmas. In Am. Geophys. Union Monogr. 13: Hart, P. J., (Ed.) p. 489-495.

Hart, S. R., 1971. K, Rb, Cs, Sr, and $\mathrm{Ba}$ contents and $\mathrm{Sr}$ isotope ratios of ocean floor basalts: Phil. Trans. Roy. Soc. London Sec. A, v. 268, p. 573-587.

Hart, S. R., Glassley, W. E., and Karig, D. E., 1972. Basalts and sea-floor spreading behind the Mariana island arc: Earth Planet. Sci. Lett. v. 15, p. 12-18. 
TABLE 5 - Continued

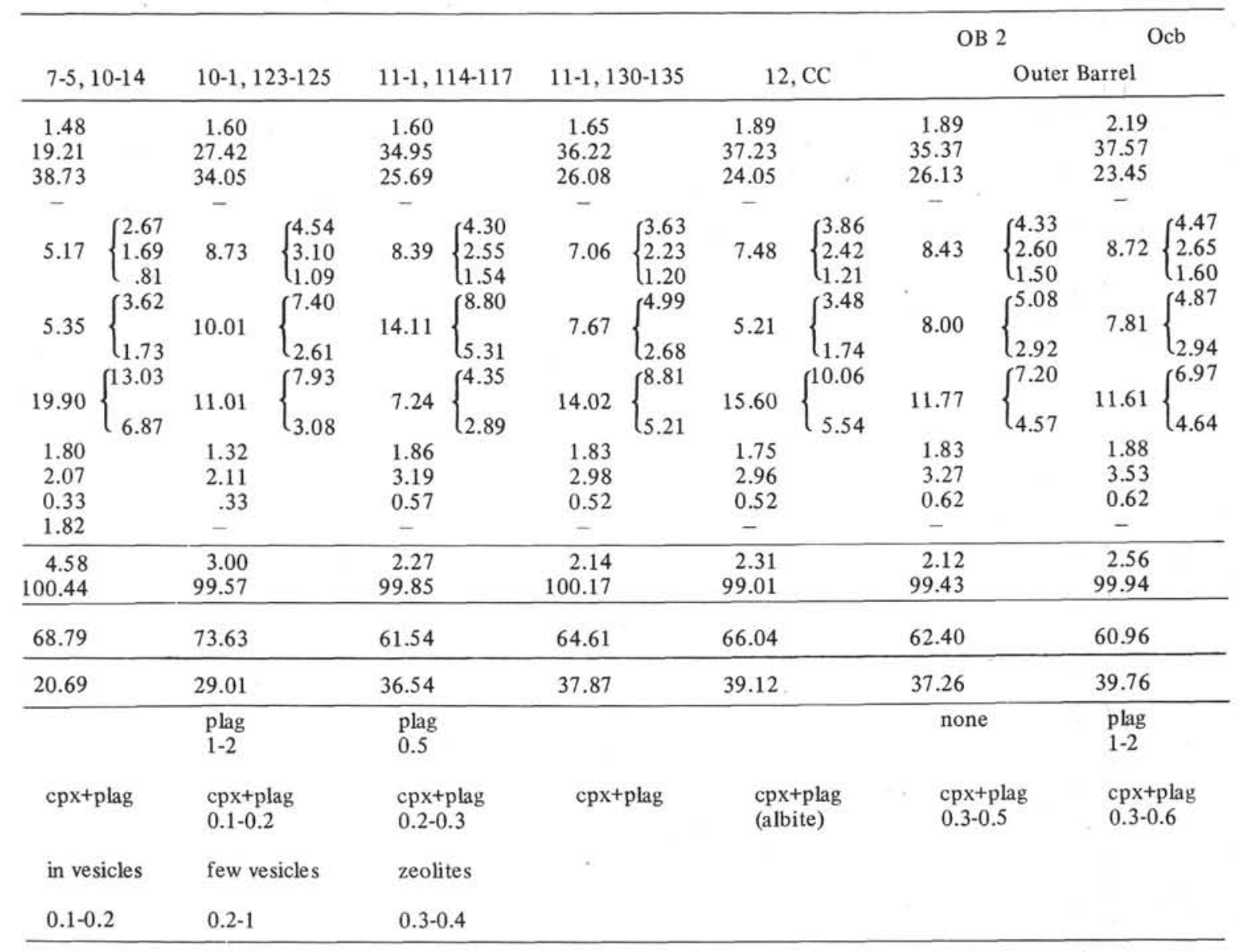

Hawkins, J. W., 1976. Petrology and geochemistry of basaltic rocks of the Lau basin: Earth Planet. Sci. Lett. v. 28, p. 283-297.

Heezen, B. C., Gray, C., Segre, A. G., and Zarndzki, E. F. K., 1971. Evidence of foundered continental crust beneath the Central Tyrrhenian Sea: Nature v. 229, p. 327-329.

Honnorez, J., and Keller, J., 1968. Xenolithe in vulkanischen Gesteinen der Aeolischen Inseln: Geol. Rdschau v. 57, p. 719-736.

Karig, D. E., 1971. Origin and development of marginal basins in the Western Pacific: J. Geophys. Res. v. 76, p. 2542-2561.

Kay, R., Hubbard, N. J., and Gast, P. W., 1970. Chemical characteristics and origin of oceanic ridge volcanic rocks: J. Geophys. Res., v. 75, p. 1985-1613.

Keller, J., 1974. Petrology of some volcanic rock series of the Aeolian arc, Southern Tyrrhenian Sea: Calcalkaline and shoshonitic associations: Contrib. Mineral. Petrol., v. 46, p. 29-47.

Keller, J., and Leiber, J., 1974. Sedimente, Tephralagen und Basalte der südtyrrhenischen Tiefsee-Ebene im Bereich des Marsili-Seeberges: Meteor. Forschung. v. 19, p. 62-76.

Kreuzer, H., Mohr, M., and Wendt, I., 1977. Potassiumargon age determination of Leg 42A, drillhole 373A, Core 7, basalt samples: this volume.

Lowrie, W. and Alvarez, W., 1974. Rotation of the Italian Peninsula: Nature v. 251, p. 285-288.

Maccarone, E., 1970. Notizie petrografiche e petrochimiche sulle lave sottomarine del Seamount 4 (Tirreno Sud): Boll. Soc. Geol. Ital., v. 89, p. 159-180.

Menard, H. W., 1967. Transitional types of crust nuclei small ocean basins: J. Geophys. Res. v. 72, p. 3061-3073.
Miyashiro, A., Shido., F., and Ewing, M., 1971. Metamorphism in the Mid-Atlantic Ridge near $24^{\circ}$ and $30^{\circ} \mathrm{N}$ : Phil. Trans. R oy. Soc. London Sec. A, v. 268, p. 589-603.

Miyashiro, A., 1975. Volcanic rock series and tectonic setting: An. Rev. Earth Planet. Sci., v. 3, p. 251-269.

Ogniben, L., Martinis, B., Rossi, P. M., Fuganti, A., Pasquare, G., Sturani, C., Nardi, R., Cocozza, T., Praturlon, A ., Parotto, M., d'Argenio, B., Pescatore, T., Scandone, P., Vezzani, L., AGIP-Attivita Minerarie, Finetti, I., Morelli, C., Caputo, M., Postpichel, D. and Giese, P., 1973. Modello strutturale d'Italia, 1:1000 000: Consiglio nazionale delle richerche, Roma.

Puchelt, H., Emmermann, R ., and Srivastava, K., 1976. Rare earth and other trace elements in basalts from the MidAtlantic Ridge $36^{\circ}$ N., DSDP Leg 37: In Aumento, F. Melson, W. G., et al., Initial Reports of the Deep Sea Drilling Project, Volume 37: Washington (U.S. Government Printing Office), p. 581-590.

Ridley, W. I., Rhodes, J. M., Reid, A. M., Jakes, P., Shih, C., and Bass, N. M., 1974. Basalts from leg 6 of DSDP: J. Petrol., v. 15 , p. $140-159$.

Ritsema, A. R., 1970. On the origin of western Mediterranean sea basins: Tectonophysics, v. 10, p. 609-623.

Robinson, P. T. and Whitford D. J., 1974. Basalts from the Eastern Indian Ocean, DSDP Leg 27. In Heirtzler, J., Veevers, J., et al., Initial Reports of the Deep Sea Drilling Project Volume 27: Washington (U.S. Government Printing Office), p. 551-559.

Ryan, W. B. F., Stanley, D. J., Hersey, J. B., Fahlquist, D. A., and Allan, T. D., 1970. The tectonics and geology of the Mediterranean Sea. In Maxwell, A. F. (Ed.), The sea, v.4, p. 387-492. 

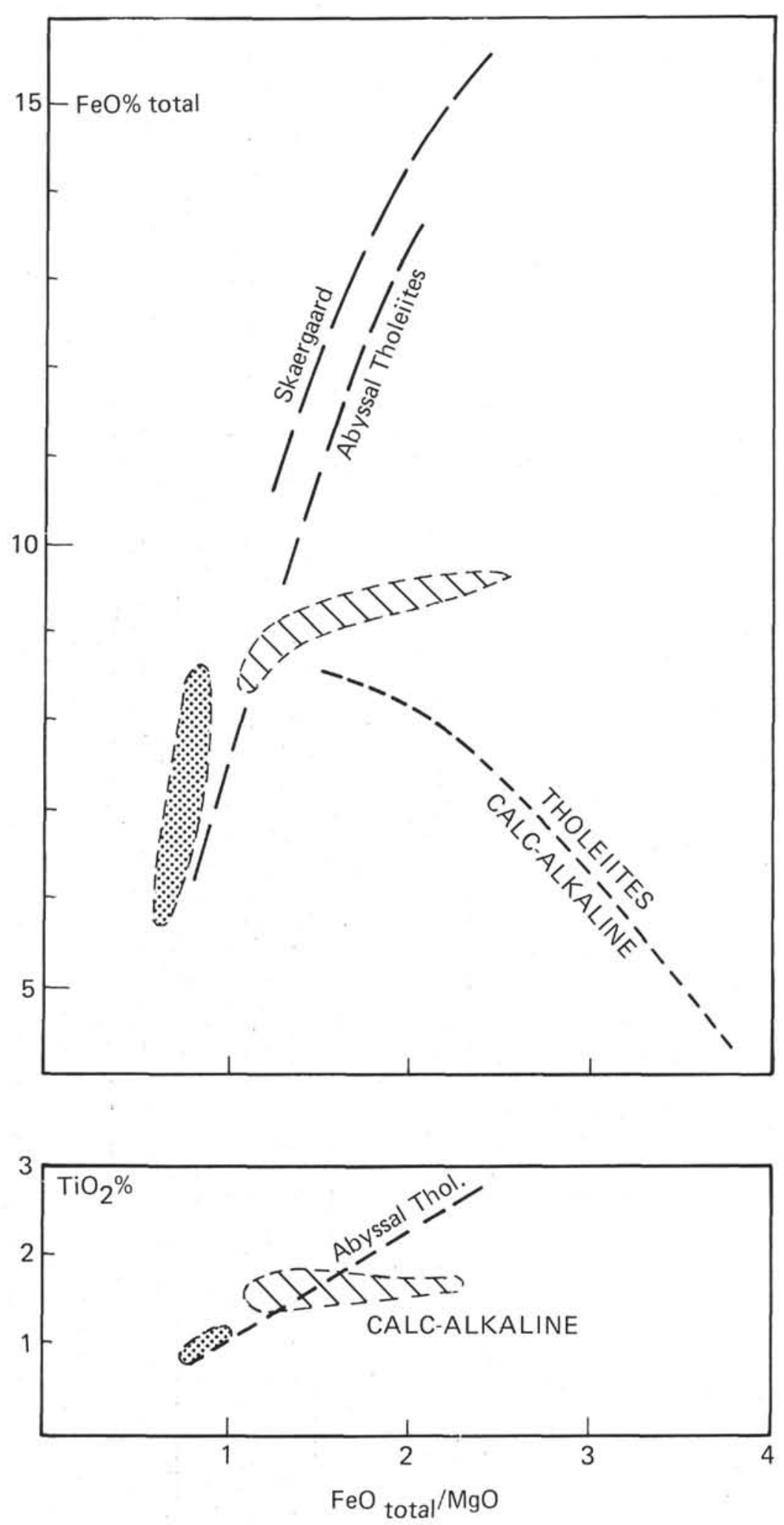

Figure 10. $\mathrm{FeO}_{\text {Total }}$ and $\mathrm{TiO}_{2}$ versus $\mathrm{FeO}_{\text {Total }} / \mathrm{MgO}$ of the Hole $373 \mathrm{~A}$ basalts. The $\mathrm{FeO}_{\text {Total }} / \mathrm{MgO}$ ratio indicates the degree of fractional crystallization (after Miyashiro, 1975). Stippled area $=$ low-Ti basalts; hachured area $=$ high-Ti basalts.

Shido, F., Miyashiro, A., and Ewing, M., 1971. Crystallization of abyssal tholeiites: Contrib. Mineral. Petrol., v. 31, p. 251-266.
Schilling, J. G., 1971. Sea floor evolution: rare earth evidence: Phil. Trans. Roy. Soc. London, Ser. A, v. 268, p. 663-706. 

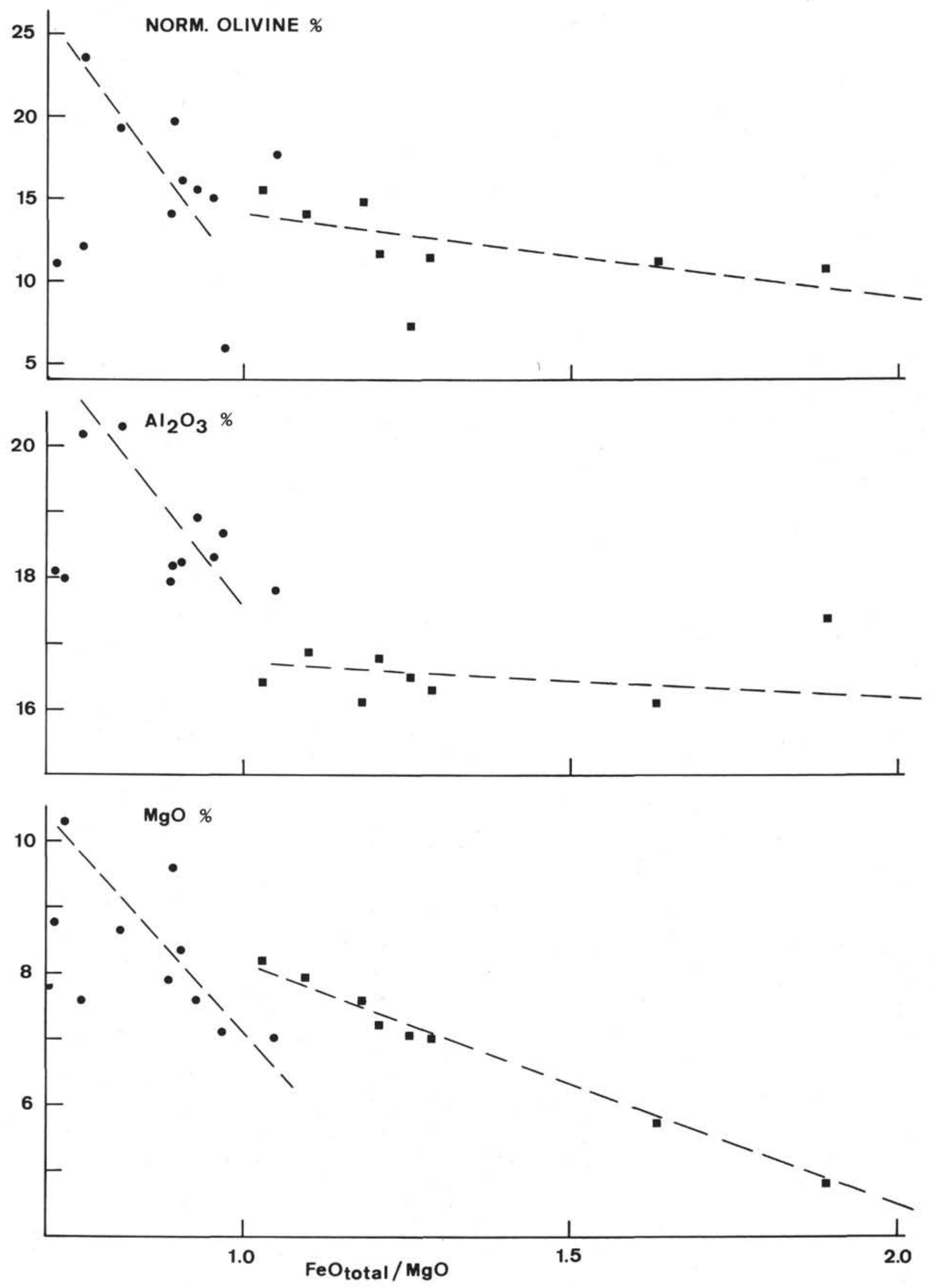

Figure 11. $\mathrm{MgO}, \mathrm{Al}_{2} \mathrm{O}_{3}$, and normative olivine contents versus $\mathrm{FeO}$ Total/MgO. Trend of compositional variation is indicated by straight lines. 
V. DIETRICH, R. EMMERMANN, H. PUCHELT, J. KELLER
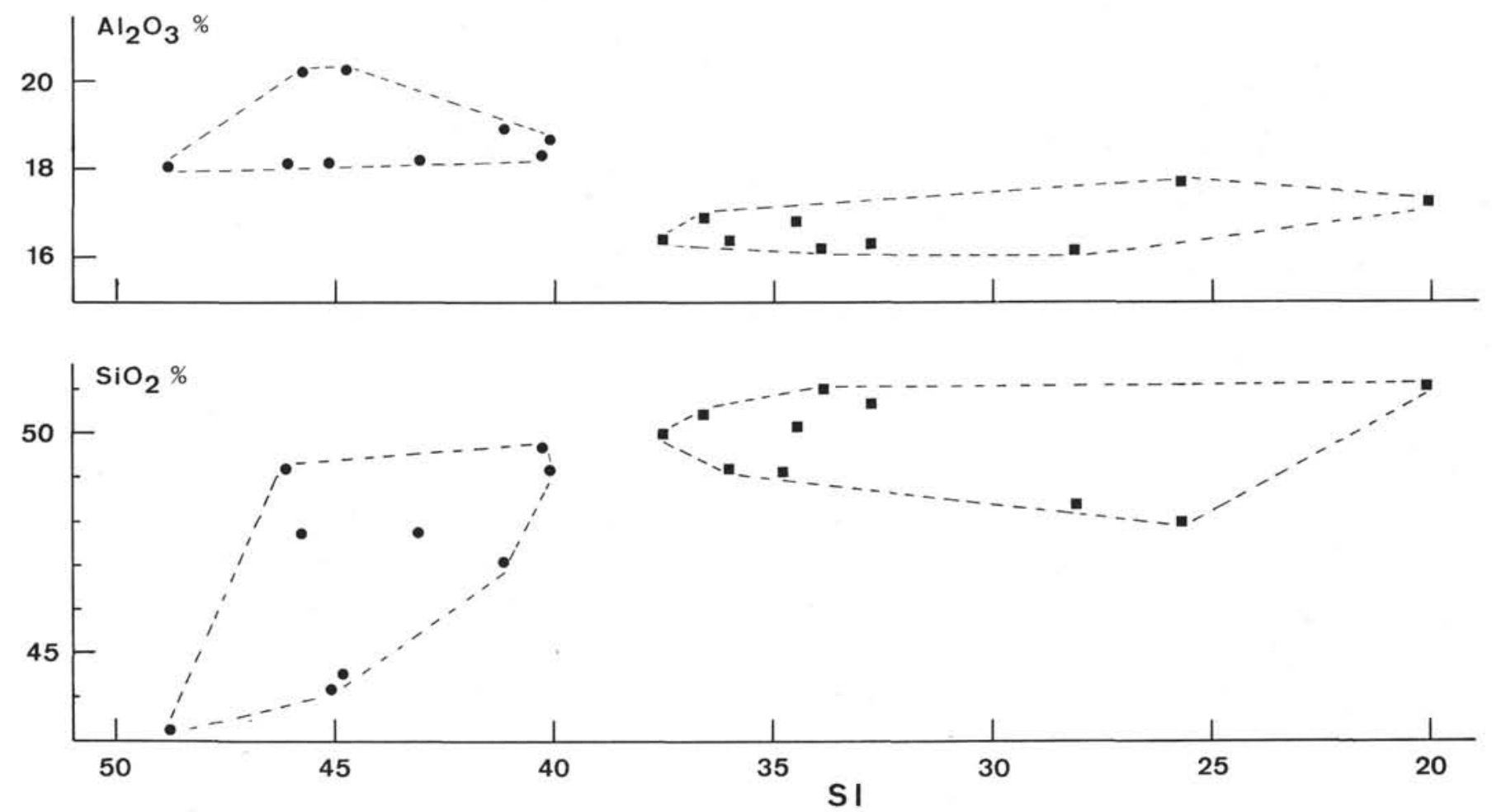

Figure 12. Oxide percents plotted against the solidification index $\left.(\mathrm{SI})=\mathrm{MgO} / \mathrm{MgO}+\mathrm{FeO}+0.9 \mathrm{Fe}_{2} \mathrm{O}_{3}+\mathrm{Na}_{2} \mathrm{O}+\mathrm{K}_{2} \mathrm{O}\right) 100$.

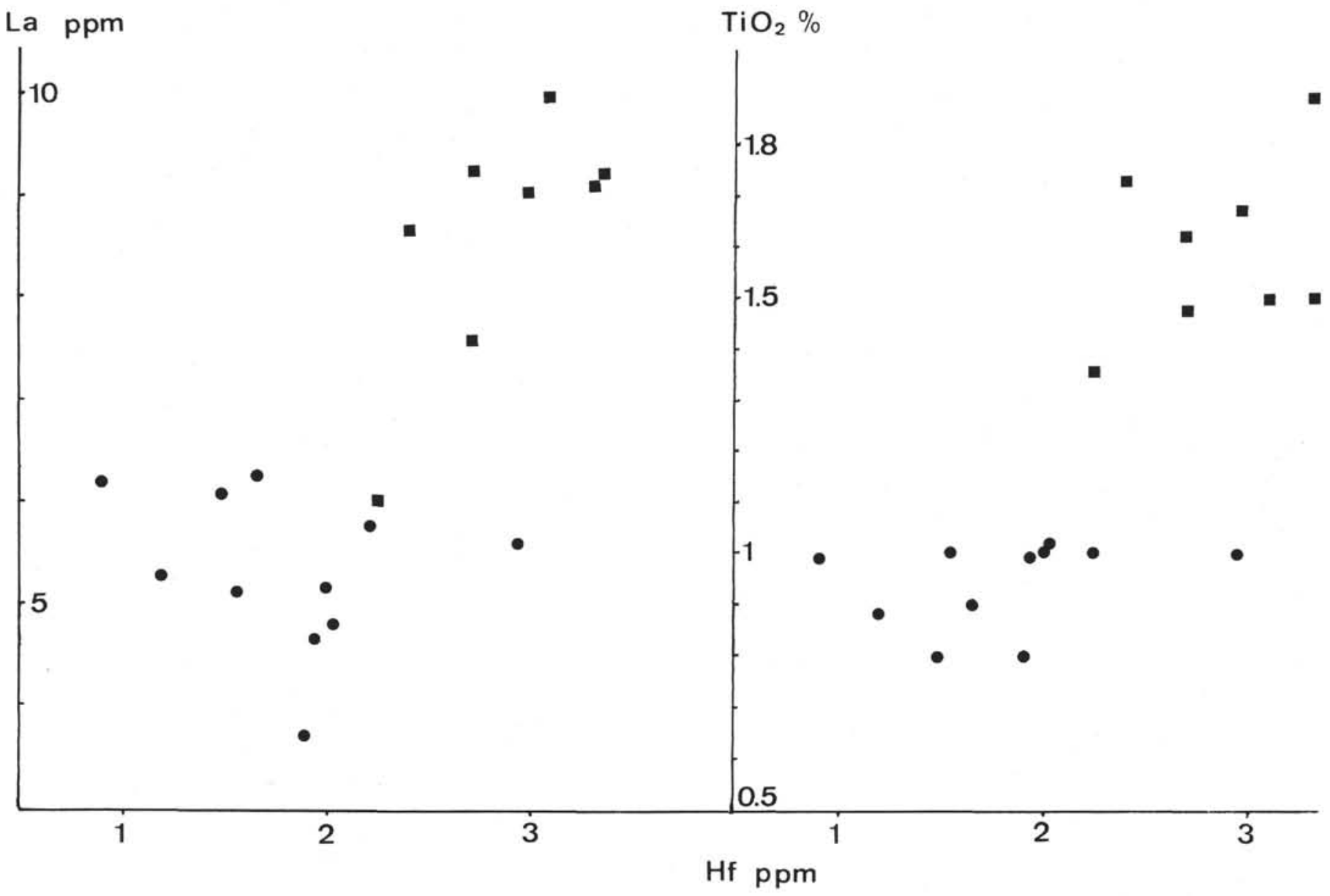

Figure 13. La/Hf and $\mathrm{TiO}_{2} \mathrm{Hf}$ plots for Hole $373 \mathrm{~A}$. 

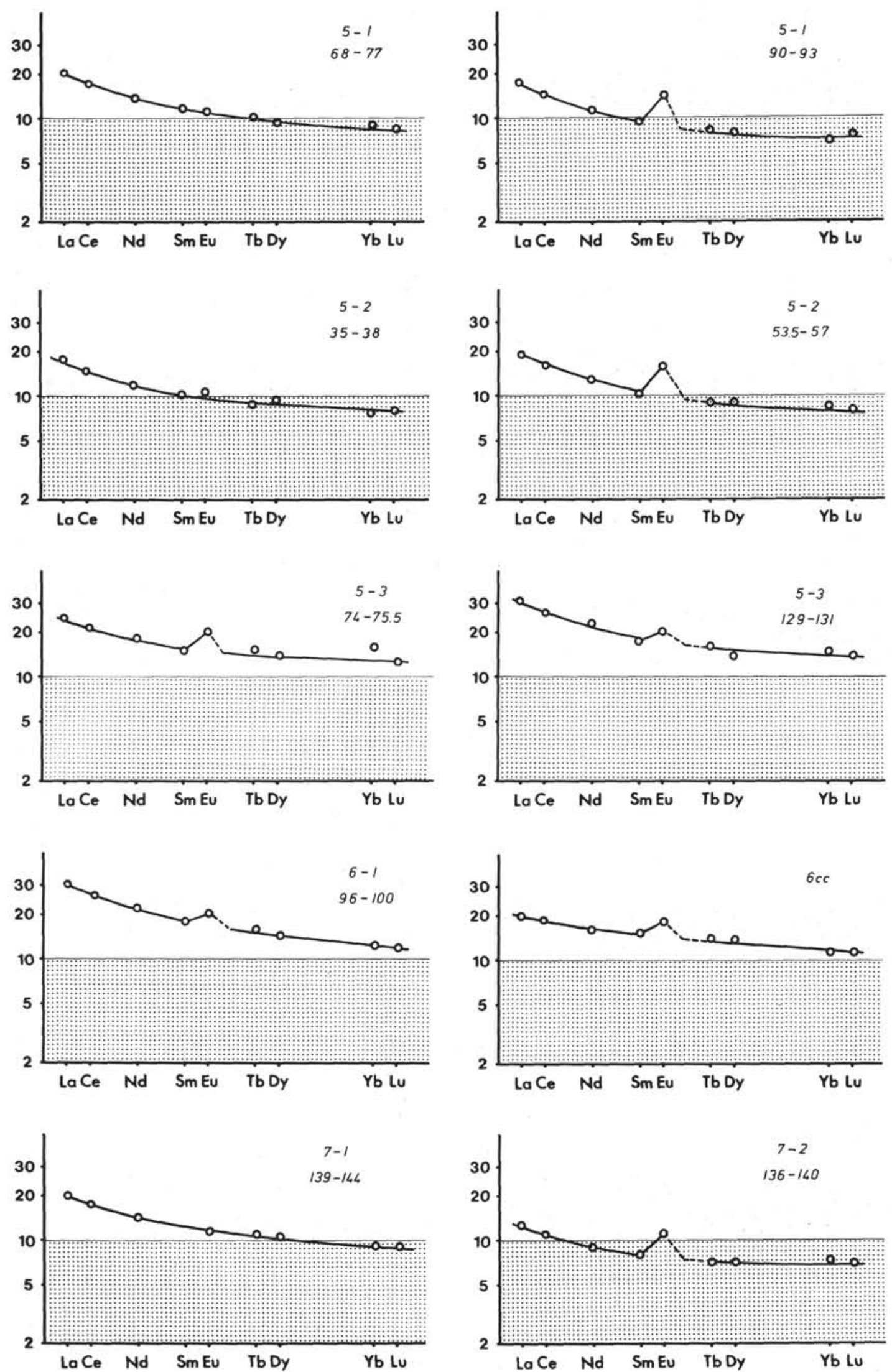

Figure 14. REE pattern of the 20 basaltic samples, Hole $373 \mathrm{~A}$. 

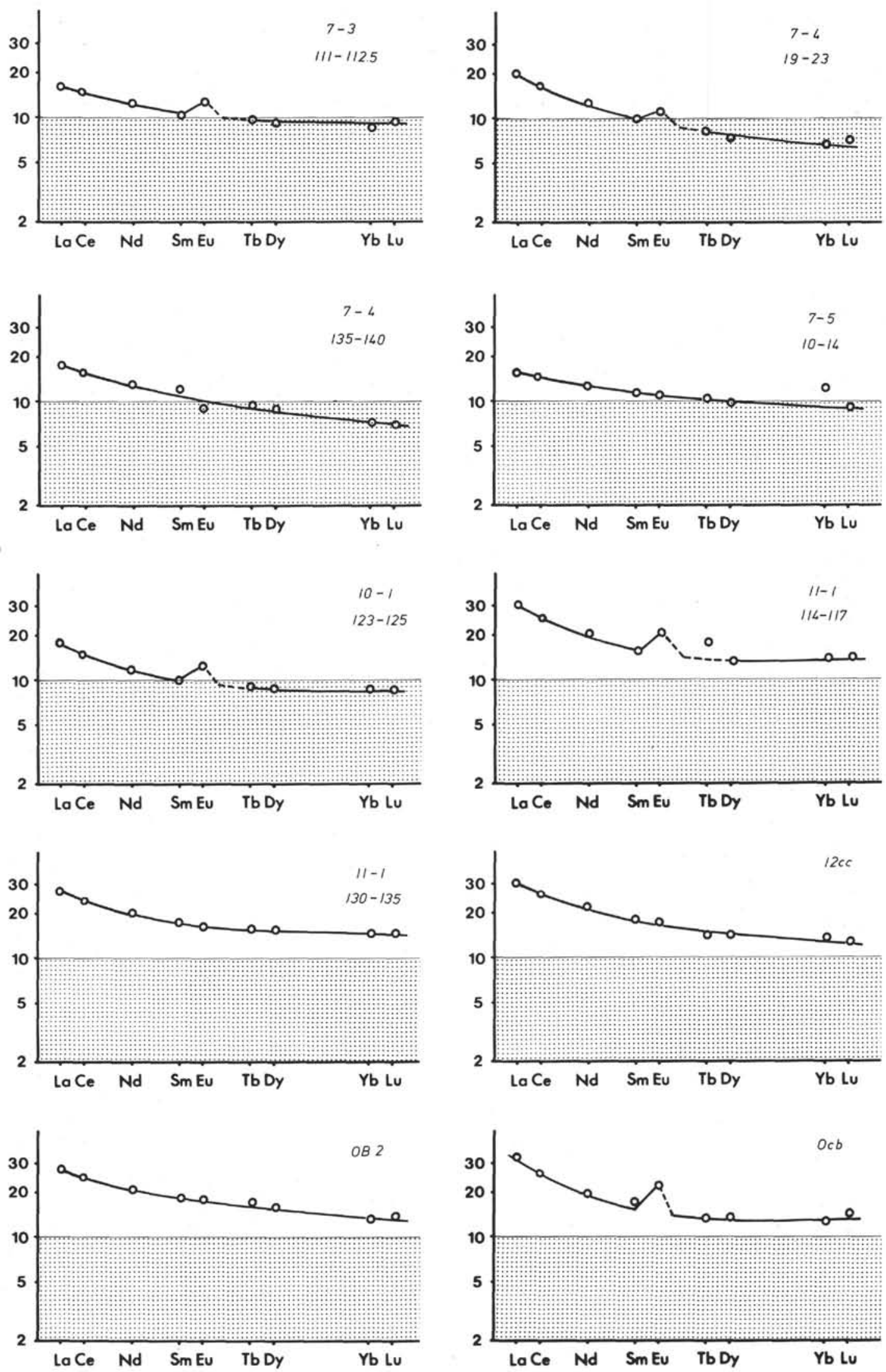

Figure 14. (Continued). 